\title{
A Peridynamic Material Model for the Analysis of Dynamic Crack Propagation in Orthotropic Media
}

\author{
M. Ghajari ${ }^{\mathrm{a}}$, L. Iannucci ${ }^{\mathrm{b}}$, P. Curtis ${ }^{\mathrm{c}}$ \\ ${ }^{a}$ Department of Aeronautics, Imperial College London \\ South Kensington Campus, London, SW7 2AZ, UK \\ m.ghajari@imperial.ac.uk \\ Tel: + 442075945107 \\ ${ }^{\mathrm{b}}$ Department of Aeronautics, Imperial College London \\ South Kensington Campus, London, SW7 2AZ, UK \\ lo.iannucci@imperial.ac.uk \\ ${ }^{c}$ Defence Science and Technology Laboratory, Salisbury, Wiltshire, SP4 0JQ, UK \\ ptcurtis@dstl.gov.uk
}

\begin{abstract}
A new material model for the dynamic fracture analysis of anisotropic materials has been proposed within the framework of the bond-based peridynamic theory. This model enables predicting complex fracture phenomena such as spontaneous crack nucleation and crack branching, curving and arrest, a capability inherited from the bond-based peridynamic theory. An important feature of the model is that the bond properties, i.e. the stiffness constant and critical stretch, are continuous functions of bond orientation in the principal material axes. This facilitates fracture analysis of anisotropic materials with random orientations, such as polycrystalline microstructures. Elastic and fracture behaviour of the model has been verified through simulating uniaxial tension of a composite plate and fracture of a cortical bone compact tension specimen, and making quantitative comparisons to analytical and experimental data. To further demonstrate the capabilities of the proposed model, dynamic fracture of a polycrystalline microstructure (alumina ceramic) has been simulated. The influence of the grain boundary and grain interior fracture energies on the interacting and competing fracture modes of polycrystalline materials, i.e. intergranular and transgranular fracture, has been studied.
\end{abstract}

Keywords: peridynamics, anisotropy, fracture, bone, ceramic

\section{Introduction}

Optimum design of most engineering structures requires accurate prediction of the fracture behaviour of materials. In the continuum mechanics theory, several techniques have been proposed and implemented in numerical programs to solve complex fracture problems. Much attention has been paid to isotropic materials, whose stiffness and strength properties are not direction-dependent. Prediction of fracture propagation in anisotropic media, such as 
composites, ceramics, rocks and bone, is also of great technological and clinical importance. However, this problem is more complicated because direction-dependence of stiffness and strength properties should be included in the formulation.

In order to predict fracture in anisotropic materials, researchers have extended numerical methods established for isotropic materials. Boone et al. (1987) used the Finite Element Method (FEM) to simulate crack propagation in unidirectional (UD) fibre-reinforced composites and in rocks. Aliabadi and Sollero (1998) proposed a 2D anisotropic material model suitable for implementation in a Boundary Element Method (BEM) code. The model was used to simulate crack propagation in UD fibre-reinforced composites (Aliabadi and Sollero 1998) and in rocks (Chen et al. 1998; Ke et al. 2009). Motamedi and Mohammadi (2010) further developed the extended FEM (X-FEM) to simulate crack propagation in 2D anisotropic media, such as UD fibre-reinforced composite plies. In the approaches mentioned above, the maximum circumferential tensile stress theory is often used to estimate the crack propagation path. For a material with anisotropic fracture properties, the circumferential stress needs to be normalised with respect to the fracture properties; the crack grows in the direction where the normalised circumferential tensile stress is maximum. These methods also require quite complex laws to predict crack nucleation and branching, which are mostly developed for isotropic materials (Belytschko et al. 2003).

The peridynamic theory of continuum mechanics was proposed by (Silling 2000) to overcome some of the intrinsic limitations of the classical continuum mechanics theory when dealing with problems that contain discontinuous displacement fields, such as fracture problems. In this theory, each infinitesimal unit of the continuum, called particle, interacts with other particles located in its neighbourhood through forces, similar to the molecular dynamics theory (Parks et al. 2008; Parks et al. 2010). The peridynamic theory is nonlocal since the interaction between particles extends beyond their immediate neighbourhood.

Anisotropic peridynamic models have been proposed by (Xu et al. 2008; Hu et al. 2012; Oterkus and Madenci 2012) to simulate crack propagation in UD fibre-reinforced composites. These models have been able to provide predictions of fibre/matrix fracture and delamination, which had good qualitative agreement with experimental observations. However, the models proposed in (Xu et al. 2008) and (Oterkus and Madenci 2012) require a uniform grid of particles and can only be used to model plies with a certain orientation. Hu et al. (Hu et al. 2012) proposed a method to remove these limitations, but it significantly adds to the computational cost for problems that use a non-uniform grid or a uniform grid with an arbitrary orientation of fibres. Another anisotropic peridynamic model was briefly described 
in Askari et al. (2008) without presenting relevant equations, where the results of crack propagation in a polycrystalline microstructure were presented. In this paper, a new $2 \mathrm{D}$ anisotropic material model suitable for the bond-based peridynamic theory has been proposed. This model can be used with any discretisation of the domain. Several fracture problems are solved with this model and quantitative comparisons are made with analytical or experimental data.

This paper is organised as follows. The bond-based peridynamic theory is briefly reviewed in section 2 . The proposed material model is introduced in section 3 followed by a description of the numerical implementation in section 4. Using the proposed model, three example problems are solved in section 5, where convergence of the solutions is discussed and the results are compared with experimental or analytical data. Some concluding remarks are presented in section 6 .

\section{Bond-based peridynamic theory}

In the bond-based peridynamic theory (Silling 2000), the equation of motion of a particle at position $\mathbf{x}$ in the reference configuration is written as:

$$
\rho \ddot{\mathbf{u}}(\mathbf{x}, t)=\int_{H_{\mathbf{x}}} \mathbf{f}\left(\mathbf{u}\left(\mathbf{x}^{\prime}, t\right)-\mathbf{u}(\mathbf{x}, t), \mathbf{x}^{\prime}-\mathbf{x}\right) d V_{\mathbf{x}^{\prime}}+\mathbf{b}(\mathbf{x}, t)
$$

where $\rho$ is the mass density, $\mathbf{u}$ is the displacement vector field, $\mathbf{f}$ is a pairwise force function, which is the force per volume squared that the particle $\mathbf{x}^{\prime}$ exerts on the particle $\mathbf{x}$, and $\mathbf{b}$ is the body force vector field. $H_{\mathbf{x}}$ is a finite volume surrounding $\mathbf{x}$, referred to as the neighbourhood of $\mathbf{x}$. It is usually taken to be a sphere in $3 \mathrm{D}$ or a circle in $2 \mathrm{D}$ problems, centred at $\mathbf{x}$ (Figure 1). Its radius, called the horizon, is denoted by $\delta$. In order to ensure conservation of linear momentum and angular momentum, the following relations should hold:

$$
\begin{array}{rr}
\mathbf{f}(\boldsymbol{\eta}, \xi)=-\mathbf{f}(-\boldsymbol{\eta},-\xi) & \forall \boldsymbol{\eta}, \boldsymbol{\xi} \\
(\boldsymbol{\eta}+\xi) \times \mathbf{f}(\boldsymbol{\eta}, \boldsymbol{\xi})=0 & \forall \boldsymbol{\eta}, \boldsymbol{\xi}
\end{array}
$$

where

$$
\xi=\mathbf{x}^{\prime}-\mathbf{x}
$$




$$
\boldsymbol{\eta}=\mathbf{u}\left(\mathbf{x}^{\prime}, t\right)-\mathbf{u}(\mathbf{x}, t)
$$

$\xi$ is the relative position vector in the reference configuration and $\boldsymbol{\eta}$ is the relative displacement vector. The vector $\xi$ is called a bond. According to eqs. (2) and (3), the forces that two particles exert on each other are equal in magnitude and opposite in direction, and they are parallel to the relative position vector in the current configuration.

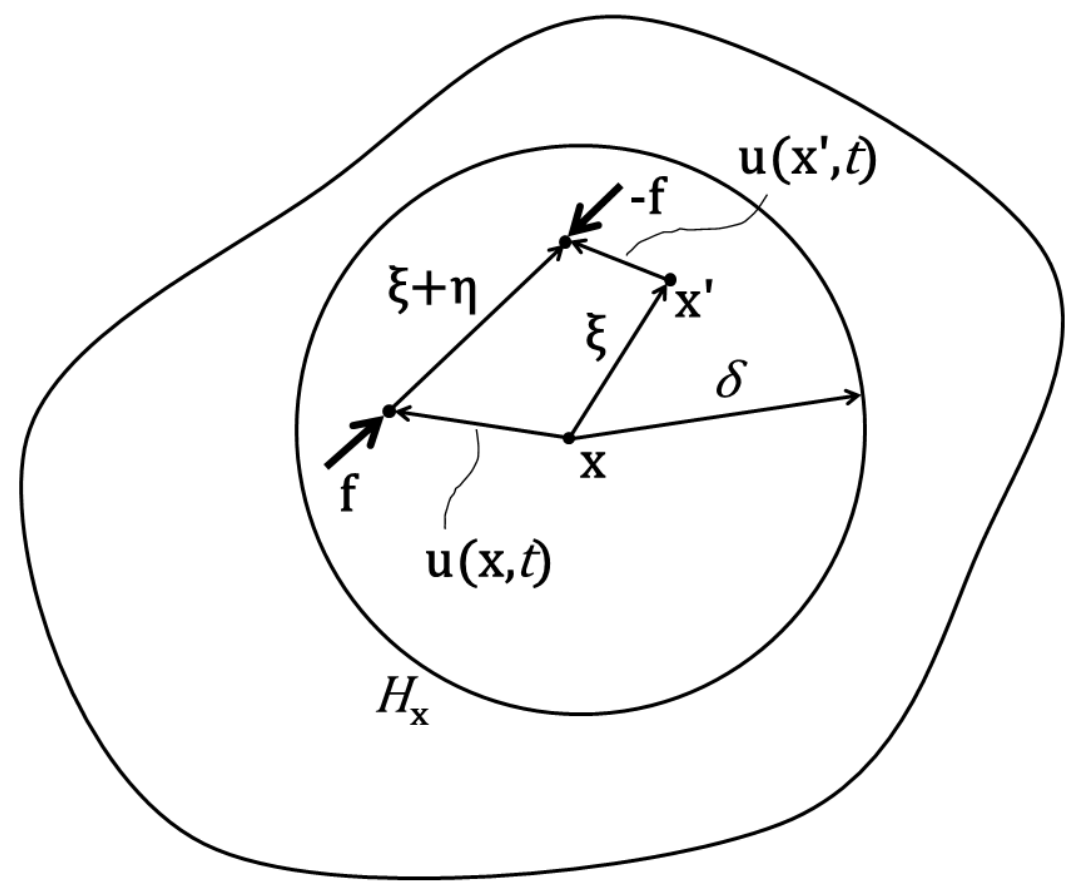

Figure 1 Illustration of peridynamic variables; the horizon is shown in the reference configuration.

In the peridynamic equation of motion, eq. (1), no spatial derivative appears, in contrast to the equation of motion of the classical theory. $\mathbf{f}$ is a function of relative displacements, which contains the constitutive equation. For a microelastic material (Silling 2000), the pairwise force function is derivable from a scalar-valued function $w(\boldsymbol{\eta}, \xi)$, called the pairwise potential function or micropotential in (Silling and Askari 2005), such that:

$$
\mathbf{f}(\boldsymbol{\eta}, \boldsymbol{\xi})=\frac{\partial w}{\partial \boldsymbol{\eta}}(\boldsymbol{\eta}, \xi) \quad \forall \boldsymbol{\eta}, \boldsymbol{\xi}
$$

Silling and Askari (2005) proposed a prototype microelastic brittle (PMB) material with the following pairwise force function: 


$$
\mathbf{f}(\boldsymbol{\eta}, \xi)=c(\xi) s(\boldsymbol{\eta}, \xi) \mu(\mathbf{x}, t, \xi) \frac{\boldsymbol{\eta}+\xi}{\|\boldsymbol{\eta}+\xi\|} \quad \forall \boldsymbol{\eta}, \xi
$$

In this equation, $c(\xi)$ is the bond stiffness constant, analogous to a spring constant, and $s$ is the bond stretch defined by:

$$
s(\boldsymbol{\eta}, \xi)=\frac{\|\boldsymbol{\eta}+\xi\|-\|\xi\|}{\|\xi\|}
$$

In eq. (7), $\mu$ determines the failure condition of the bond. It is one for an intact bond. If the bond stretch exceeds a critical value $\left(s_{0}(\xi)\right), \mu$ becomes zero permanently, implementing breakage of the bond.

The relation between the bond stiffness constant and the elastic modulus is determined by setting equal the strain energy density obtained from the peridynamic theory for a given loading condition and the strain energy density obtained from the classical theory of elasticity for the same loading condition. For a microelastic material, the strain energy density at a particle is the integral of the micropotential over the neighbourhood. For a particle with a neighbourhood fully contained within a body, i.e. far from surfaces or interfaces (Silling and Askari 2005):

$$
W^{P D}=\frac{1}{2} \int_{H_{\mathbf{x}}} w(\boldsymbol{\eta}, \xi) d V_{\mathbf{x}^{\prime}}
$$

For the PMB material:

$$
w(\boldsymbol{\eta}, \xi)=\frac{c(\xi) s^{2} \xi}{2}
$$

where, $\xi:=\|\xi\|$. Gerstle et al. (2005) have shown that for an isotropic material under planestress or plane-strain conditions:

$$
c=\frac{12 E}{\pi \delta^{3} t(1+v)}
$$

where $t$ is the thickness of the structure, and 
plane-stress: $v=1 / 3$, plane-strain: $v=1 / 4$.

As can be seen, this model leads to constant Poisson's ratio. In other words, the model requires only one material constant to be fully defined, while the elastic behaviour of isotropic materials is defined with two independent material constants. This is a consequence of the assumptions made for the bond-based peridynamic theory (Silling and Askari 2005; Hu et al. 2012; Oterkus and Madenci 2012). This limitation has been removed in the state-based peridynamic theory (Silling et al. 2007), where the interaction between $\mathbf{x}$ and $\mathbf{x}^{\prime}$ depends on the collective behaviour of their neighbouring particles. The material model presented in this paper was developed within the bond-based peridynamic framework.

\section{Transversely isotropic material model}

Anisotropy can be included in the bond-based peridynamic theory by varying bond properties with the direction of $\xi$. Proper selection of this dependency simplifies the evaluation of the integrals that will appear when trying to obtain the relation between bond properties and engineering constants, i.e. the integrations of eqs. (26), (36) and (37), and also results in a desirable behaviour of the material. The direction of $\xi$ in the principal material frame (the 123 frame shown in Figure 2) can be described with the polar coordinate, $\varphi \in$ $[0, \pi]$, and the azimuthal coordinate, $\theta \in[0,2 \pi)$. According to the convention used for most transversely isotropic materials (materials that have a plane, called the plane of isotropy, in which the mechanical properties are equal in all directions, such as UD composites), the 1 axis is taken to be normal to the plane of isotropy. A few terms of the following expansion may be used to define the dependency of the bond stiffness constant on the direction of the bond: 


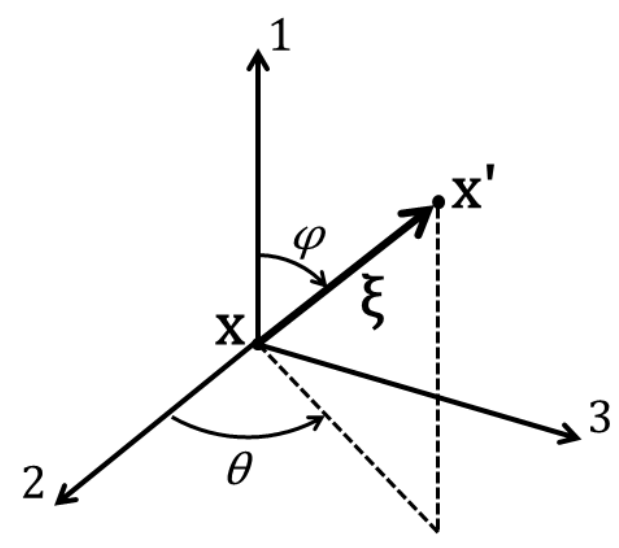

Figure 2 Direction of a peridynamic bond in the principal material axes.

$$
c(\varphi, \theta)=\sum_{n=0}^{\infty} \sum_{m=0}^{n} P_{n}^{m}(\cos \varphi)\left(A_{n m} \cos m \theta+B_{n m} \sin m \theta\right),
$$

where $P_{n}^{m}$ are the associated Legendre functions of degree $n$ and order $m$ :

$$
P_{n}^{m}(\cos \varphi)=\frac{(-1)^{m}}{2^{n} n !}\left(1-\cos ^{2} \varphi\right)^{\frac{m}{2}} \frac{d^{n+m}}{d(\cos \varphi)^{n+m}}\left(\cos ^{2} \varphi-1\right)^{n}
$$

and $A_{n m}$ and $B_{n m}$ are constant coefficients (MacRobert 1967). Eq. (13) is the spherical harmonic expansion of the bond stiffness constant. For a transversely isotropic material, one may assume that the bond stiffness constant, $c$, is not a function of $\theta$. This means that $A_{n m}=B_{n m}=0$ for $m>0$ in eq. (13), which leads to a simplification of this equation as:

$$
c(\varphi, \theta)=c(\varphi)=\sum_{n=0}^{\infty} A_{n 0} P_{n}^{0}(\cos \varphi)
$$

For a transversely isotropic material, one may also assume that $c(\varphi)$ is symmetrical with respect to the 23 plane (plane of isotropy), i.e. $c(\varphi)=c(\pi-\varphi)$. Using the property of the associated Legendre functions $P_{n}^{0}(-\cos \varphi)=(-1)^{n} P_{n}^{0}(\cos \varphi)$, one then concludes that $A_{n 0}=0$ for $=1,3,5, \ldots$. Hence, the spherical harmonic expansion of $c$ up to the eighth degree will be: 


$$
\begin{aligned}
c(\varphi)=A_{00}+ & A_{20} P_{2}^{0}(\cos \varphi)+A_{40} P_{4}^{0}(\cos \varphi)+A_{60} P_{6}^{0}(\cos \varphi) \\
& +A_{80} P_{8}^{0}(\cos \varphi)
\end{aligned}
$$

where

$$
\begin{gathered}
P_{2}^{0}(\cos \varphi)=\frac{1}{2}\left(3 \cos ^{2} \varphi-1\right), \\
P_{4}^{0}(\cos \varphi)=\frac{1}{8}\left(35 \cos ^{4} \varphi-30 \cos ^{2} \varphi+3\right), \\
P_{6}^{0}(\cos \varphi)=\frac{1}{16}\left(231 \cos ^{6} \varphi-315 \cos ^{4} \varphi+105 \cos ^{2} \varphi-5\right), \\
P_{8}^{0}(\cos \varphi)=\frac{1}{128}\left(6435 \cos ^{8} \varphi-12012 \cos ^{6} \varphi+6930 \cos ^{4} \varphi-1260 \cos ^{2} \varphi\right. \\
+35) .
\end{gathered}
$$

This form of $c$ is suitable for integration. The same model will be used in section 3.2 to define the dependency of the critical stretch, $s_{0}$, on $\varphi$.

In order to fully determine $c(\varphi)$ in eq. (16), its value should be known for five different angles. For the 1 and 2 axes, one may assume $c(0)=c_{1}$ and $c\left(90^{\circ}\right)=c_{2} \cdot c_{1}$ and $c_{2}$ are constants of the material model, which are determined by the material properties as explained in section 3.1. $c(\varphi)>0$ is a sufficient, but not necessary, condition for the material model to be stable (Silling et al. 2003). Hence, several oscillations of $c(\varphi)$ on $[0, \pi / 2]$, including negative and positive values, may be theoretically possible but in order to achieve numerical stability and convergence, the domain must be finely discretised, which will significantly increase the computational cost. Therefore, the three remaining conditions, required to fully determine $c(\varphi)$, should be chosen with a view to obtain a smooth transition of $c(\varphi)$ between 0 and $\pi / 2$, particularly for cases with $c_{1} / c_{2} \gg 1$ (such as the example problems presented in sections 5.1 and 5.2). In this study, it was assumed that $c(\varphi)=c_{2}$ for $\varphi=45^{\circ}, 55^{\circ}$ and $65^{\circ}$. The polar plot of $c(\varphi)$ for $c_{1} / c_{2}=10$, shown in Figure 3, illustrates the intended smooth transition (this plot will be further discussed in the next paragraph). In addition, the relatively rapid convergence of the numerical solution for a UD composite (highly anisotropic material), presented in section 5.1, confirmed the suitability of the assumptions. By solving the system of five linear equations, the following relations were obtained for the constant coefficients:

$$
\begin{gathered}
A_{00}=0.0722 c_{1}+0.9278 c_{2}, \\
A_{20}=0.3021\left(c_{1}-c_{2}\right), \\
A_{40}=0.3376\left(c_{1}-c_{2}\right), \\
A_{60}=0.2159\left(c_{1}-c_{2}\right),
\end{gathered}
$$




$$
A_{80}=0.0722\left(c_{1}-c_{2}\right)
$$

It is should be noted that when the material is isotropic, i.e. $c_{1}=c_{2}, c=A_{00}=c_{1}$, which does not depend on $\varphi$.

In (Seleson et al. 2013), a model for anisotropic materials, in the context of nonlocal diffusion, was presented. Following this reference:

$$
c(\varphi)=\frac{\xi \cdot \mathbf{K} \xi}{\|\xi\|^{2}}
$$

where $\mathbf{K}$ is a second-order tensor. In two dimensions, using polar coordinates $\left(\xi_{1}=\xi \cos \varphi\right.$ and $\left.\xi_{2}=\xi \sin \varphi\right)$, eq. (19) can be written as:

$$
\begin{aligned}
c(\varphi) & =\frac{\xi_{1}^{2} K_{11}+\xi_{1} \xi_{2}\left(K_{12}+K_{21}\right)+\xi_{2}^{2} K_{22}}{\xi^{2}} \\
& =\cos ^{2} \varphi K_{11}+\cos \varphi \sin \varphi\left(K_{12}+K_{21}\right)+\sin ^{2} \varphi K_{22}
\end{aligned}
$$

Again assuming that $c(\varphi)$ is symmetrical with respect to the plane of isotropy (the 23 plane in Figure 2), and that $c(0)=c_{1}$ and $c\left(90^{\circ}\right)=c_{2}$ lead to $K_{12}+K_{21}=0, K_{11}=c_{1}$ and $K_{22}=c_{2}$; thus:

$$
c(\varphi)=c_{2}+\left(c_{1}-c_{2}\right) \cos ^{2} \varphi
$$

This equation is similar to the one suggested by (Silling and Askari 2005) to include anisotropy in the microelastic response. Eq. (21) can also be derived from the spherical harmonic expansion of $c$ up to the second degree. From eqs. (15) and (17):

$$
c(\varphi)=A_{00}+A_{20} P_{2}^{0}(\cos \varphi)=A_{00}+A_{20} \frac{1}{2}\left(3 \cos ^{2} \varphi-1\right) .
$$

Assuming $c(0)=c_{1}$ and $c\left(90^{\circ}\right)=c_{2}$, it can be shown that eq. (22) leads to eq. (21). Therefore, the model presented in this paper is a generalisation of the models proposed in (Silling and Askari 2005; Seleson et al. 2013) for anisotropic materials. Figure 3 shows polar plots of $c(\varphi)$ represented with spherical harmonics up to the second degree, eq. (21), and up to the eighth degree, eq. (16), for two different ratios of $c_{1} / c_{2}$. As can be seen, using eq. (16) results in significantly stronger anisotropy in the distribution of $c(\varphi)$, while maintaining the 
smooth transition of $c(\varphi)$ on $[0, \pi / 2]$. Strong anisotropy in the distribution of $c(\varphi)$ and $s_{0}(\varphi)$ is a desirable property, which allows for modelling materials with various levels of anisotropy in stiffness and strength. A quantitative comparison between the toughness anisotropies achievable with these models is presented in section 3.2.

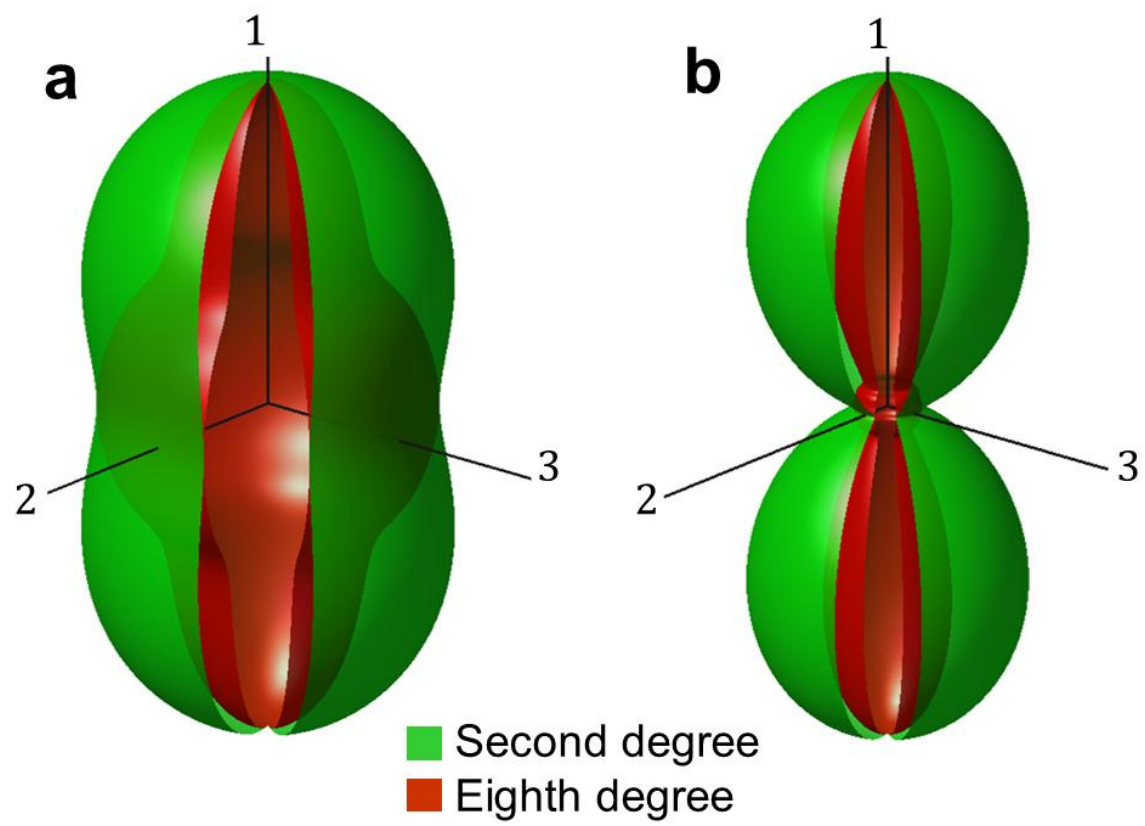

Figure 3 Polar plots of the bond stiffness constant represented with spherical harmonics up to the second and the eighth degrees; a) $c_{2}=0.5 c_{1}$ and b) $c_{2}=0.1 c_{1}$.

\subsection{Bond stiffness constant}

In order to obtain the relation between the bond stiffness constants, $c_{1}$ and $c_{2}$, and elastic moduli, the approach introduced by (Gerstle et al. 2005) was used. Under a certain strain state, the strain energy density of a particle was determined from the peridynamic formulation and it was set equal to the strain energy determined from the classical theory of elasticity. In the classical theory of elasticity, the stress-strain relations (Hooke's law) for an orthotropic material under plane-stress or plane-strain conditions in the 12 plane (Figure 2) can be written, in the principal material axes and using the Voigt notation, as:

$$
\left[\begin{array}{c}
\sigma_{1} \\
\sigma_{2} \\
\tau_{12}
\end{array}\right]=\left[\begin{array}{ccc}
C_{11} & C_{12} & 0 \\
C_{12} & C_{22} & 0 \\
0 & 0 & C_{66}
\end{array}\right]\left[\begin{array}{c}
\varepsilon_{1} \\
\varepsilon_{2} \\
\gamma_{12}
\end{array}\right]
$$


where $C_{i j}$ is the stiffness matrix. The strain energy density of an element under $\varepsilon_{i}$ strain state is:

$$
W^{C L}=\frac{1}{2} C_{i j} \varepsilon_{i} \varepsilon_{j}
$$

where $i$ and $j=1,2,6$ and $\varepsilon_{6}=\gamma_{12}$. To derive the relations between the bond stiffness constants and the constants of the stiffness matrix, a system of four equations were formed by assuming four different strain states. The strain states and corresponding peridynamic bond stretches are presented in Table 1 . To obtain the bond stretch, the strain vector $\left[\varepsilon_{1}, \varepsilon_{2}, \gamma_{12}\right]^{T}$ was transformed to a coordinate system whose first axis was aligned with the bond. In the new coordinate system, the first component of the strain tensor was the bond stretch. For example, if the new coordinate system is $\mathrm{xy}$ and the angle from the 1 axis to the $\mathrm{x}$ axis is $\varphi$, then:

$$
s=\varepsilon_{1} \cos ^{2} \varphi+\varepsilon_{2} \sin ^{2} \varphi+\gamma_{12} \cos \varphi \sin \varphi
$$

Table 1 Strain states and the corresponding stretch.

\begin{tabular}{|l|l|c|}
\hline $\mathrm{n}$ & Strain state & Bond stretch \\
\hline 1 & $\varepsilon_{1}=\zeta$ and other components are zero & $s=\zeta \cos ^{2} \varphi$ \\
\hline 2 & $\varepsilon_{2}=\zeta$ and other components are zero & $s=\zeta \sin ^{2} \varphi$ \\
\hline 3 & $\gamma_{12}=\zeta$ and other components are zero & $s=\zeta \cos \varphi \sin \varphi$ \\
\hline 4 & $\varepsilon_{1}=\varepsilon_{2}=\zeta$ and other components are zero & $s=\zeta$ \\
\hline
\end{tabular}

Under plane-stress or plane-strain conditions, the strain energy density of a particle with a neighbourhood fully contained within a body is:

$$
W^{P D}=\frac{1}{2} \int_{0}^{2 \pi} \int_{0}^{\delta}\left[\frac{c(\varphi) s^{2} \xi}{2}\right] \xi t d \xi d \varphi .
$$

The system of four equations $W_{n}^{C L}-W_{n}^{P D}=0, n=1 \ldots 4$ (see Table 1) was solved, which resulted in:

$$
c_{1}=\frac{15.41 C_{11}-7.41 C_{22}}{\pi \delta^{3} t}
$$




$$
\begin{gathered}
c_{2}=\frac{8.08 C_{22}-0.08 C_{11}}{\pi \delta^{3} t}, \\
C_{12}=C_{66}=0.059 C_{11}+0.274 C_{22} .
\end{gathered}
$$

For an isotropic material, $C_{11}=C_{22}$, and eqs. (27) and (29) simplify to:

$$
\begin{gathered}
c_{1}=\frac{8 C_{11}}{\pi \delta^{3} t}, \\
C_{12}=C_{66}=0.333 C_{11} .
\end{gathered}
$$

The stiffness matrices for an isotropic material under plane stress and plane strain conditions are:

$$
\begin{gathered}
\text { plane stress: } \frac{E}{1-v^{2}}\left[\begin{array}{ccc}
1 & v & 0 \\
v & 1 & 0 \\
0 & 0 & \frac{1-v}{2}
\end{array}\right], \\
\text { plane strain: } \frac{E}{(1+v)(1-2 v)}\left[\begin{array}{ccc}
1-v & v & 0 \\
v & 1 & 0 \\
0 & 0 & \frac{1-2 v}{2}
\end{array}\right] .
\end{gathered}
$$

Substituting the components of the stiffness matrix from eqs. (32) and (33) into eqs. (30) and (31) leads to the same results obtained by Grestle (2005), i.e. eqs. (11) and (12).

The proposed anisotropic material model allows only two elastic constants, $c_{1}$ and $c_{2}$, while the stiffness matrix in eq. (23) has four constants. Hence, the assumptions of the bondbased peridynamic model impose restrictions on two constants of the stiffness matrix, i.e. eq. (29), and as a result, the stiffness matrix has only two independent constants. These restrictions can probably be removed within the state-based peridynamic framework, where the response of a particle depends on the collective behaviour of all particles in its neighbourhood rather than the deformation of the pair-wise bonds between the particle and its neighbours. This should be investigated in future works.

\subsection{Critical bond stretch}

The spherical harmonic expansion was also used to define the dependency of the critical bond stretch on the direction of $\xi$ : 


$$
\begin{aligned}
s_{0}^{2}(\varphi) & =B_{00}+B_{20} P_{2}^{0}(\cos \varphi)+B_{40} P_{4}^{0}(\cos \varphi)+B_{60} P_{6}^{0}(\cos \varphi) \\
& +B_{80} P_{8}^{0}(\cos \varphi) .
\end{aligned}
$$

The constant coefficients were obtained by using the same assumptions leading to eq. (18), as follows:

$$
\begin{gathered}
B_{00}=0.0722 s_{01}^{2}+0.9278 s_{02}^{2}, \\
B_{20}=0.3021\left(s_{01}^{2}-s_{02}^{2}\right), \\
B_{40}=0.3376\left(s_{01}^{2}-s_{02}^{2}\right), \\
B_{60}=0.2159\left(s_{01}^{2}-s_{02}^{2}\right), \\
B_{80}=0.0722\left(s_{01}^{2}-s_{02}^{2}\right) .
\end{gathered}
$$

$S_{01}$ is the critical stretch of the bonds at $\varphi=0$ and $s_{02}$ is the critical stretch of the bonds at $\varphi=90^{\circ}$.

The relation between the bond critical stretch and the mode I critical strain energy release rate was determined by using the method introduced in (Silling and Askari 2005). According to this method, the energy required for a crack to split a body into two halves equals the sum of the rupture energy of the bonds that initially crossed the crack surface. The critical strain energy release rate is this energy divided by the area of the crack surface. This method can be applied to brittle materials, where other dissipative mechanisms, as compared with fracture, are negligible. The critical strain energy release rates for mode I crack propagation in the planes normal to the 1 and 2 axes, $G_{I c 1}$ and $G_{I c 2}$ respectively, can be determined from the following integrals:

$$
\begin{gathered}
G_{I C 1}=\int_{0}^{\delta} \int_{\mathrm{z}}^{\delta} \int_{-\cos ^{-1}\left(\frac{z}{\xi}\right)}^{\cos ^{-1}\left(\frac{z}{\xi}\right)}\left[\frac{c(\varphi) s_{0}^{2}(\varphi) \xi}{2}\right] t \xi \mathrm{d} \varphi \mathrm{d} \xi \mathrm{d} z \\
G_{I c 2}=\int_{0}^{\delta} \int_{z}^{\delta} \int_{\sin ^{-1}\left(\frac{z}{\xi}\right)}^{\pi-\sin ^{-1}\left(\frac{z}{\xi}\right)}\left[\frac{c(\varphi) s_{0}^{2}(\varphi) \xi}{2}\right] t \xi \mathrm{d} \varphi \mathrm{d} \xi \mathrm{d} z .
\end{gathered}
$$

The variables of the integrals are defined in Figure 4. Representation of $c$ and $s$ with a few terms of the spherical harmonics expansion facilitates evaluation of these integrals. The integrals were evaluated with Mathematica 8.0 (Wolfram 2010), leading to:

$$
\mathrm{G}_{\mathrm{Ic} 1}=\mathrm{t} \delta^{4}\left(0.063 \mathrm{c}_{1} \mathrm{~s}_{01}^{2}+0.022 \mathrm{c}_{1} \mathrm{~s}_{02}^{2}+0.022 \mathrm{c}_{2} \mathrm{~s}_{01}^{2}+0.144 \mathrm{c}_{2} \mathrm{~s}_{02}^{2}\right)
$$




$$
\mathrm{G}_{\mathrm{Ic} 2}=\mathrm{t} \delta^{4}\left(0.010 \mathrm{c}_{1} \mathrm{~s}_{01}^{2}+0.008 \mathrm{c}_{1} \mathrm{~s}_{02}^{2}+0.008 \mathrm{c}_{2} \mathrm{~s}_{01}^{2}+0.224 \mathrm{c}_{2} \mathrm{~s}_{02}^{2}\right)
$$

Assuming isotropic properties, it can be shown that:

$$
s_{01}^{2}=s_{02}^{2}=\frac{4 G_{I}}{c t \delta^{4}}
$$

which is identical to the relation obtained by (Gerstle et al. 2005) for isotropic materials under plane-stress or plane-strain conditions. Finally, the critical stretches of the bonds were determined as:

$$
\begin{aligned}
\mathrm{s}_{01}^{2} & =\frac{500\left[\left(4 \mathrm{G}_{\mathrm{Ic} 1}-11 \mathrm{G}_{\mathrm{Ic} 2}\right) \mathrm{c}_{1}+\left(112 \mathrm{G}_{\mathrm{Ic} 1}-72 \mathrm{G}_{\mathrm{Ic} 2}\right) \mathrm{c}_{2}\right]}{\mathrm{t} \delta^{4}\left(71 \mathrm{c}_{1}^{2}+3168 \mathrm{c}_{1} \mathrm{c}_{2}+944 \mathrm{c}_{2}^{2}\right)} \\
\mathrm{s}_{02}^{2} & =\frac{500\left[\left(31.5 \mathrm{G}_{\mathrm{Ic} 2}-5 \mathrm{G}_{\mathrm{Ic} 1}\right) \mathrm{c}_{1}+\left(11 \mathrm{G}_{\mathrm{Ic} 2}-4 \mathrm{G}_{\mathrm{Ic} 1}\right) \mathrm{c}_{2}\right]}{\mathrm{t} \delta^{4}\left(71 \mathrm{c}_{1}^{2}+3168 \mathrm{c}_{1} \mathrm{c}_{2}+944 \mathrm{c}_{2}^{2}\right)}
\end{aligned}
$$

$s_{01}^{2}$ and $s_{02}^{2}$ cannot be less than zero otherwise bond rupture generates energy. This restricts the $G_{I c 1} / G_{I c 2}$ ratio. For a material with isotropic stiffness properties $\left(c_{1}=c_{2}\right)$,

$$
0.72<G_{I c 1} / G_{I c 2}<4.7
$$

which are the consequences of eqs. (41) and (42) with the assumptions of $s_{01}^{2}>0$ and $s_{02}^{2}>0$. Cold drawn steel is an example of a material that has isotropic stiffness properties but anisotropic fracture toughness (Toribio and Ayaso 2003). It should be noted that the fracture behaviour of the proposed material model is fully defined by using the mode I fracture energies. This implies that the mode II (sliding) fracture energy of the model is not independent from its mode I fracture energies, which is another consequence of the assumptions of the bond-based peridynamic theory.

If eq. (21) is used to define $c(\varphi)$, and $s_{0}^{2}(\varphi)$ so that

$$
s_{0}^{2}(\varphi)=s_{02}^{2}+\left(s_{01}^{2}-s_{02}^{2}\right) \cos ^{2} \varphi
$$

integrals (36) and (37) lead to 


$$
\begin{aligned}
& s_{01}^{2}=\frac{2\left(G_{I c 1}-G_{I c 2}\right) c_{1}+\left(8 G_{I c 1}-3 G_{I c 2}\right) c_{2}}{5 t \delta^{4}\left(2 c_{1}^{2}+11 c_{1} c_{2}+2 c_{2}^{2}\right)}, \\
& s_{02}^{2}=\frac{\left(8 G_{I c 2}-3 G_{I c 1}\right) c_{1}+2\left(G_{I c 2}-G_{I c 1}\right) c_{2}}{5 t \delta^{4}\left(2 c_{1}^{2}+11 c_{1} c_{2}+2 c_{2}^{2}\right)} .
\end{aligned}
$$

Again for a material with isotropic stiffness properties $\left(c_{1}=c_{2}\right)$,

$$
0.5<G_{I c 1} / G_{I c 2}<2
$$

which are the consequences of eqs. (45) and (46) with the assumptions of $s_{01}^{2}>0$ and $s_{02}^{2}>0$. The upper limit of $G_{I c 1} / G_{I c 2}$ in eq. (43) is significantly greater than that in eq. (47). This is due to stronger anisotropy in the distribution of $s_{0}^{2}(\varphi)$, which was obtained by using more terms of the spherical harmonic expansion to define the dependency of $s_{0}$ on $\varphi$, as compared with eq. (44). Obviously, this model allows for modelling materials with higher degrees of anisotropy in toughness, such as bone (section 5.2).
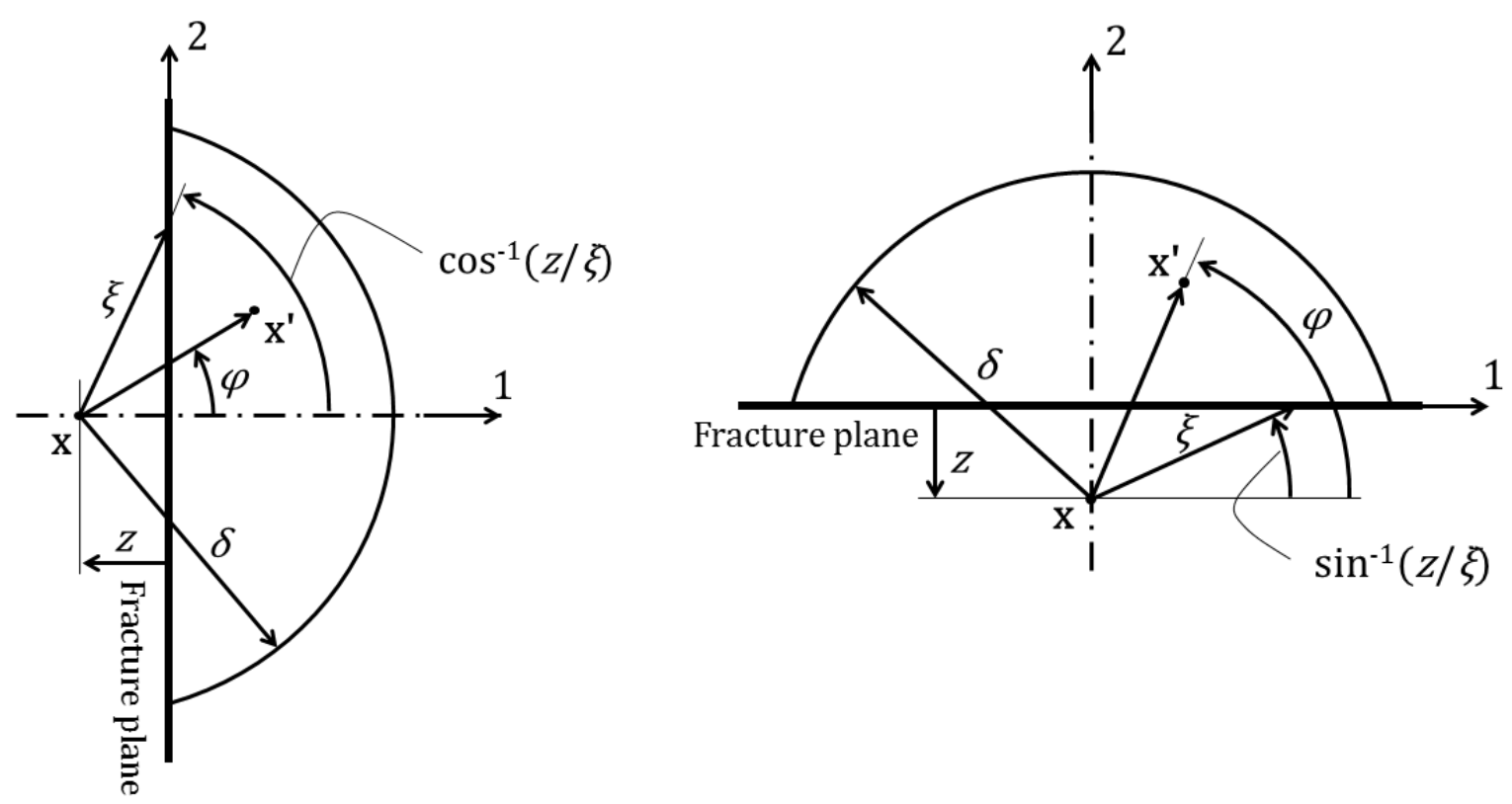

Figure 4 Definition of variables used for determination of the strain energy release rates (left) $G_{I c 1}$ and (right) $G_{I c 2}$.

For the PMB material, damage at each particle has been defined (Silling and Askari 2005) as the number of broken bonds to the total number of bonds: 


$$
d=1-\frac{\int_{H_{\mathbf{x}}} \mu(\mathbf{x}, t, \xi) d V_{\mathbf{x}^{\prime}}}{\int_{H_{\mathbf{x}}} d V_{\mathbf{x}^{\prime}}} .
$$

This definition does not include any information about possible dependence of failure properties of bonds on their orientation. Hence, it is suitable for isotropic materials, such as the PMB material. In the proposed anisotropic model, bonds have different rupture energies depending on their orientation. For this model, a suitable definition of damage, based on the rupture energy of the failed bonds, would be:

$$
d=1-\frac{\int_{H_{\mathbf{x}}}\left[\frac{c(\varphi) s_{0}^{2}(\varphi) \xi}{2}\right] \mu(\mathbf{x}, t, \xi) d V_{\mathbf{x}^{\prime}}}{\int_{H_{\mathbf{x}}}\left[\frac{c(\varphi) s_{0}^{2}(\varphi) \xi}{2}\right] d V_{\mathbf{x}^{\prime}}}
$$

$d$ can change from 0 , when all bonds are intact, to 1 , when all bonds are broken. If isotropic properties are assumed, eq. (49) simplifies to:

$$
d=1-\frac{\int_{H_{\mathbf{x}}} \xi \mu(\mathbf{x}, t, \xi) d V_{\mathbf{x}^{\prime}}}{\int_{H_{\mathbf{x}}} \xi d V_{\mathbf{x}^{\prime}}}
$$

which is not identical to eq. (48) because eq. (48) was defined based on the number, and not the rupture energy, of bonds. In this paper, eq. (49) was used to define damage at each particle.

\section{Numerical implementation}

In order to solve complex problems with the peridynamic theory, a numerical approach should be adopted. The approach used in this study, was first described in (Silling and Askari 2005). According to this approach, the continuum region is discretised into nodes, which together form a grid. Each node has a finite volume. The integral in eq. (1) is replaced with a finite sum, as follows:

$$
\rho \ddot{\mathbf{u}}\left(\mathbf{x}_{i}, t_{k}\right)=\sum_{j} \mathbf{f}\left(\mathbf{u}\left(\mathbf{x}_{j}, t_{k}\right)-\mathbf{u}\left(\mathbf{x}_{i}, t_{k}\right), \mathbf{x}_{j}-\mathbf{x}_{i}\right) V_{j}+\mathbf{b}\left(\mathbf{x}_{i}, t_{k}\right)
$$


where $V_{j}$ is the volume of node $j$ and $\mathbf{f}$ is supplied from eq. (7). The sum is taken over all nodes $j$ that satisfy $\left\|\mathbf{x}_{j}-\mathbf{x}_{i}\right\| \leq \delta$. The method is meshfree because there is no geometrical relation between nodes. The displacement vector of node $i$ at time $t_{k+1}$ is obtained from approximating the acceleration in the above equation with an explicit central difference formula:

$$
\ddot{\mathbf{u}}\left(\mathbf{x}_{i}, t_{k}\right)=\frac{\mathbf{u}\left(\mathbf{x}_{i}, t_{k+1}\right)-2 \mathbf{u}\left(\mathbf{x}_{i}, t_{k}\right)+\mathbf{u}\left(\mathbf{x}_{i}, t_{k-1}\right)}{\Delta t^{2}}
$$

where $\Delta t$ is a constant time step. Hence:

$$
\mathbf{u}\left(\mathbf{x}_{i}, t_{k+1}\right)=\Delta t^{2} \ddot{\mathbf{u}}\left(\mathbf{x}_{i}, t_{k}\right)+2 \mathbf{u}\left(\mathbf{x}_{i}, t_{k}\right)-\mathbf{u}\left(\mathbf{x}_{i}, t_{k-1}\right)
$$

The truncation errors associated with these approximations have been discussed in (Silling and Askari 2005). When $k=0, \mathbf{u}\left(\mathbf{x}_{i}, t_{-1}\right)$ must be known to start the procedure in eq. (53) (it should be noted that $\dot{\mathbf{u}}\left(\mathbf{x}_{i}, t_{0}\right)$ and $\mathbf{u}\left(\mathbf{x}_{i}, t_{0}\right)$ are known from initial conditions). $\mathbf{u}\left(\mathbf{x}_{i}, t_{-1}\right)$ can be approximated (Cook et al. 2002) from its Taylor series expansion about $t_{0}$ and omitting powers of $\Delta t$ higher than second:

$$
\mathbf{u}\left(\mathbf{x}_{i}, t_{-1}\right)=\mathbf{u}\left(\mathbf{x}_{i}, t_{0}\right)-\Delta t \dot{\mathbf{u}}\left(\mathbf{x}_{i}, t_{0}\right)+\frac{\Delta t^{2}}{2} \ddot{\mathbf{u}}\left(\mathbf{x}_{i}, t_{0}\right)
$$

where $\ddot{\mathbf{u}}\left(\mathbf{x}_{i}, t_{0}\right)$ is obtained by evaluating eq. (51) at $k=0$.

Silling and Askari (2005) derived the following condition for the stable time step:

$$
\Delta t<\sqrt{\frac{2 \rho}{\sum_{j}\left|\mathbf{C}\left(\mathbf{x}_{j}-\mathbf{x}_{i}\right)\right| V_{j}}},
$$

where $\mathbf{C}$ is a second-order tensor and $|\mathbf{C}|=\sqrt{C_{p q} C_{p q}}$. For the PMB material, eq. (7), it can be shown that (Macek and Silling 2007):

$$
C_{p q}(\xi)=c(\xi) \frac{\xi_{p} \xi_{q}}{\left(\xi_{p} \xi_{p}\right)^{3 / 2}}
$$


where the components of the relative position vector, $\xi$, are in a Cartesian coordinate system. Relation (55) provides a necessary condition for the stable time step. For the simulations presented in the next section, the stable time step was obtained by multiplying a factor of 0.7 by the time step determined from this relation.

The model described in this paper can be used with any discretisation of the domain and an arbitrary orientation of the principal material axes. The computational model introduced in (Hu et al. 2012) for fibre-reinforced composites has the same features. However, their model has a discrete representation of bond properties, with those bonds aligned with fibre direction (fibre bonds) having properties different from all other bonds (matrix bonds). If non-uniform grid or an arbitrary fibre orientation is used, properties of each bond should be corrected with a scale factor, whose calculation adds to the computational cost. On the other hand, their model does not impose restrictions on the ratio of fracture toughness in fibre and matrix directions, in contrast to the model proposed in the current study.

Two types of convergence introduced in (Bobaru et al. 2009) were used in the current study. Assuming a uniform grid with a $\Delta x$ spacing and $\delta=m \Delta x$ :

1) the $m$-convergence is when $m$ increases while $\delta$ is fixed, and

2) the $\delta$-convergence is when $\delta$ decreases while $m$ is fixed.

A FORTRAN program has been developed to numerically solve peridynamic equations for complex problems. The pre and post-processing, e.g. discretising the domain, defining boundary and initial conditions, and visualising and manipulating the results, are performed using LS-PrePost (LSTC 2012), which is freely available.

\section{$5 \quad$ Example problems}

In this section, some problems are analysed using the proposed transversely isotropic material model in order to verify its elastic and fracture behaviour and to demonstrate its application to fracture analysis of different materials, e.g. cortical bone and polycrystalline microstructures. The problems were run on a single CPU core of a $2.66 \mathrm{GHz}$ Intel Xeon processor using 16 GB RAM. Some details of the numerical simulations are presented in Table 2. It is worth noting that for each example problem, thus given stiffness constants, the stable time step, $\Delta t$, depends on $\delta$ not $\Delta x(=\delta / m)$. 
Table 2 Some details of the numerical simulations.

\begin{tabular}{|c|c|c|c|c|c|}
\hline Example problem & $\delta[\mathrm{mm}]$ & $\mathrm{m}$ & $\begin{array}{l}\text { Approx. No. of } \\
\text { bonds }\end{array}$ & $\Delta t[\mathrm{~ns}]^{\mathrm{a}}$ & $\begin{array}{l}\text { Wall-clock run } \\
{\text { time }[\mathrm{min}]^{\mathrm{a}}}\end{array}$ \\
\hline \multirow{4}{*}{$\begin{array}{l}\text { Uniaxial tension of a } \\
\text { UD fibre-reinforced } \\
\text { composite }\end{array}$} & \multirow[t]{3}{*}{6} & 3 & 67,000 & 240 & 7 \\
\hline & & 4 & 195,000 & 240 & 25 \\
\hline & & 5 & 539,000 & 240 & 60 \\
\hline & 3 & 4 & 846,000 & 120 & 190 \\
\hline \multirow{9}{*}{$\begin{array}{l}\text { Compact tension test } \\
\text { for cortical bone }\end{array}$} & \multirow[t]{4}{*}{2} & 3 & 35,000 & 160 & 15 \\
\hline & & 4 & 102,000 & 160 & 45 \\
\hline & & 5 & 264,000 & 160 & 110 \\
\hline & & 6 & 543,000 & 160 & 230 \\
\hline & \multirow[t]{5}{*}{1} & 3 & 139,000 & 80 & 120 \\
\hline & & 4 & 420,000 & 80 & 360 \\
\hline & & 5 & $1,100,000$ & 80 & 935 \\
\hline & & 6 & $2,205,000$ & 80 & 1880 \\
\hline & & $\begin{array}{l}\text { Non-uniform } \\
\text { mesh }\end{array}$ & 520,000 & 80 & 480 \\
\hline $\begin{array}{l}\text { Dynamic fracture of } \\
\text { polycrystalline } \\
\text { microstructures }\end{array}$ & 0.010 & 5 & $4,041,000$ & 0.230 & 1500 \\
\hline
\end{tabular}

a: the values are rounded to facilitate comparisons.

\subsection{Uniaxial tension of a UD fibre-reinforced composite}

The purpose of this example is to show the accuracy of the elastic behaviour of the material model for a highly anisotropic material. The plate, shown in Figure 5, was made of a UD carbon fibre reinforced epoxy with $C_{11}=124.0 \mathrm{GPa}$ and $C_{22}=12.6 \mathrm{GPa}$. It was discretised with a uniform grid. A constant horizon $\delta=6 \mathrm{~mm}$ and different grid spacing $\Delta x=$ 1.2, 1.5 and $2 \mathrm{~mm}$ were used. The lower edge of the plate was constrained against vertical displacement. A $2 \mathrm{~mm}$ vertical displacement, ramped up over $2 \mathrm{~ms}$ to ensure quasi-static response, was applied on the upper edge. The boundary conditions were applied within a layer of $\delta$ thickness under the edge, as suggested by (Silling and Askari 2005). 


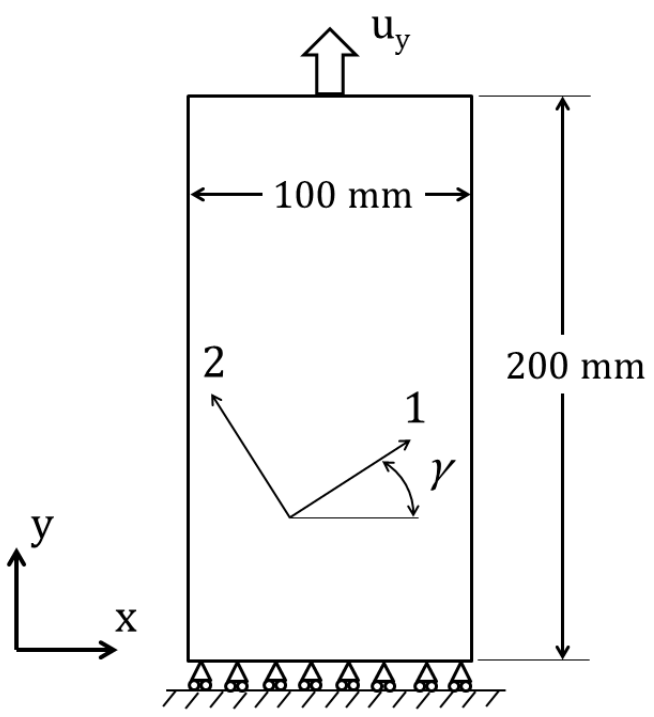

Figure 5 The anisotropic plate under tension.

The off-axis modulus, $E_{y}$, for various angles of anisotropy, $\gamma$, was determined from the simulation results and compared to its theoretical value, determined from (Jones 1999):

$$
E_{y}=\frac{1}{S_{11} \sin ^{4} \gamma+\left(2 S_{12}+S_{66}\right) \sin ^{2} \gamma \cos ^{2} \gamma+S_{22} \cos ^{4} \gamma} .
$$

where $S_{i j}$ are the components of the compliance matrix. The compliance matrix is the inverse of the stiffness matrix shown in eq. (23). To fully define the stiffness matrix, $C_{12}$ and $C_{66}$ were determined from eq. (29). It can be seen in Figure 6 that for $\gamma \leq 60^{\circ}$, the model accurately predicts the modulus with $m=3$ (approx. 67,000 bonds, Table 2). For $\gamma=90^{\circ}$, however, the peridynamic solution convergences to the theoretical result as $m$ increases to 5 (approx. 539,000 bonds). In this case, even taking $m=4$ (approx. 195,000 bonds) provides an acceptable prediction. Hence, for this highly anisotropic material, elastic behaviour can be accurately modelled with $\mathrm{m}$ values not greater than 5. It is worth mentioning that for isotropic materials, $m \geq 3$ was recommended (Silling and Askari 2005; Ha and Bobaru 2010), and for modelling anisotropic materials using a discrete representation of bond properties, $\mathrm{m}=5$ was recommended (Hu et al. 2011). 


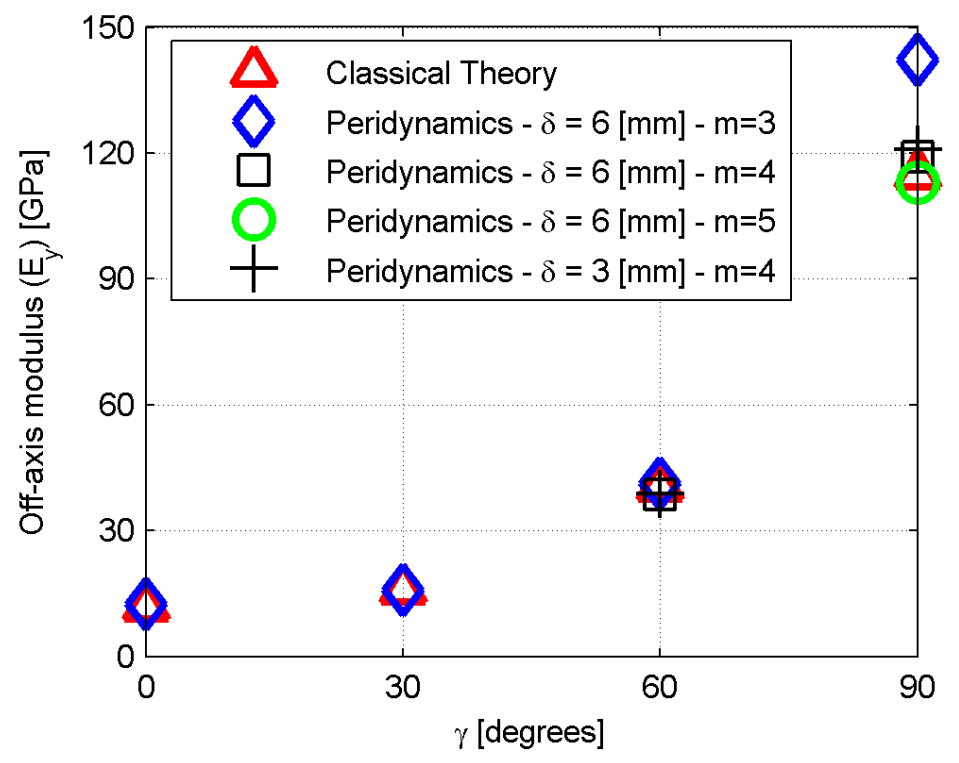

Figure 6 Off-axis modulus of the anisotropic plate vs. angle of anisotropy.

The relations between the bond stiffness constants and engineering constants, i.e. eqs. (27) and (28), were derived for a particle with a neighbourhood fully contained within a body. If these equations are used to determine the bond stiffness constants of particles that are within a $\delta$ distance from a free surface, thus having a smaller neighbourhood, their bulk elastic properties will be different from the bulk elastic properties of the particles that are inside the bulk of material. This difference, however, becomes negligible as $\delta$ decreases to zero (Ha and Bobaru 2011). In this paper, the same peridynamic parameters obtained for the particles in the bulk were used for the particles near or on the boundary.

The off-axis modulus was also predicted by using a model with a smaller horizon $\delta=3$ $\mathrm{mm}$ and $\mathrm{m}=4$ (approx. 846,000 bonds). Figure 6 shows that the prediction of this model is almost the same as the prediction of the model with $\delta=6 \mathrm{~mm}$ and $\mathrm{m}=4$, indicating that the latter model, which required significantly lower computational effort (Table 2), was good enough for the prediction of the off-axis modulus of the composite plate. It should be noted that no fracture occurred in this problem. In dynamic fracture problems where damage evolution is affected by stress waves, the horizon can influence the fracture pattern and the crack propagation speed (Ha and Bobaru 2010) because wave dispersion is dependent on the horizon (Silling 2000). Some discussions on selection of a horizon for these problems can be found in (Bobaru and $\mathrm{Hu} 2012$ ). 


\subsection{The compact tension test for cortical bone}

The compact tension test is usually used to determine the mode I critical stress intensity factor of isotropic metals. It has also been used to characterise anisotropic materials, such as fibre reinforced polymer composites (Donadon et al. 2007; Laffan et al. 2010) and cortical bone (Behiri and Bonfield 1984; Bonfield 1987; Behiri and Bonfield 1989). Crack propagation in a cortical bone compact tension specimen was predicted by using the anisotropic peridynamic material model. This test has been described in (Behiri and Bonfield 1984) and is schematically shown in Figure 7. These authors employed the compact tension test to obtain stable crack propagation so that the crack length and the corresponding load can be recorded during quasi-static loading of the specimens (Bonfield 1987).

For bovine cortical bone, $C_{11}$ and $C_{22}$ were obtained from (Van-Buskirk et al. 1981). If the 1 axis is assumed to be parallel to the anatomical axis of the bone and the 23 plane is assumed to be the plane of isotropy, $C_{11}=25 \mathrm{GPa}$ and $C_{22}=16.25 \mathrm{GPa}$. From eq. (29), $C_{12}=5.93 \mathrm{GPa}$ and $C_{66}=5.93 \mathrm{GPa}$, which are reasonably close to the measured (VanBuskirk et al. 1981) mean values of $5.89 \mathrm{GPa}$ and $6.65 \mathrm{GPa}$, respectively. As discussed before, it may be possible to develop a state-based peridynamic material model which does not impose restrictions on the constants of the stiffness matrix. Plane strain conditions were assumed since Behiri et al. (1984) showed that variation in the thickness of cortical bone compact tension specimens from $0.5 \mathrm{~mm}$ to $2 \mathrm{~mm}$ had no effect on fracture parameters. For transversely oriented bovine cortical bone $\left(\gamma=0^{\circ}\right.$ in Figure 7$)$, they measured an average critical stress intensity factor $K_{I c 2}=4.0 \mathrm{MPa} \cdot \mathrm{m}^{1 / 2}$ (crack on a plane normal to the 2 axis). By grooving cortical bone compact tension specimens, Behiri et al. (1989) determined an average $K_{I C 1}=6.5 \mathrm{MPa} \cdot \mathrm{m}^{1 / 2}$ (crack on a plane normal to the 1 axis). To define fracture parameters of the peridynamic material model, critical strain energy release rates $G_{I C 1}$ and $G_{I c 2}$ were required. They were calculated from (Sih et al. 1965):

$$
\begin{aligned}
& G_{I c 1}=K_{I c 1}^{2} \sqrt{\frac{S_{11} S_{22}}{2}} \sqrt{\sqrt{\frac{S_{11}}{S_{22}}}+\frac{2 S_{12}+S_{66}}{2 S_{22}}}, \\
& G_{I c 2}=K_{I c 2}^{2} \sqrt{\frac{S_{11} S_{22}}{2}} \sqrt{\sqrt{\frac{S_{22}}{S_{11}}}+\frac{2 S_{12}+S_{66}}{2 S_{11}}},
\end{aligned}
$$


where $S_{i j}$ are the components of the compliance matrix. The compliance matrix is the inverse of the stiffness matrix shown in eq. (23) where $C_{11}=25 \mathrm{GPa}, C_{22}=16.25 \mathrm{GPa}$ and $C_{12}=$ $C_{66}=5.93 \mathrm{GPa}$. Eq. (59) was derived by Sih et al. (1965) for orthotropic materials under plane stress or plane strain conditions in the 12 plane (Figure 2) with a crack on the 13 plane. It should be noted that in the equation presented in (Sih et al. 1965) a factor of $\pi$ appears. This factor has been included in the critical stress intensity factors reported in (Behiri and Bonfield 1984) and (Behiri and Bonfield 1989). Eq. (58) follows from eq. (59) by rotating the coordinate system by $-\pi / 2$ around the 3 axis. Using eqs. (58) and (59), and $K_{I C 1}=6.5$ MPa. $\mathrm{m}^{1 / 2}$ and $K_{I c 2}=4.0 \mathrm{MPa} \cdot \mathrm{m}^{1 / 2}, G_{I c 1}=2190 \mathrm{~J} / \mathrm{m}^{2}$ and $G_{I c 2}=1030 \mathrm{~J} / \mathrm{m}^{2}$ were determined. The latter is in good agreement with the average value of $1019 \mathrm{~J} / \mathrm{m}^{2}$, directly measured by (Behiri and Bonfield 1984). There was no direct measurement for $G_{I c 1}$.

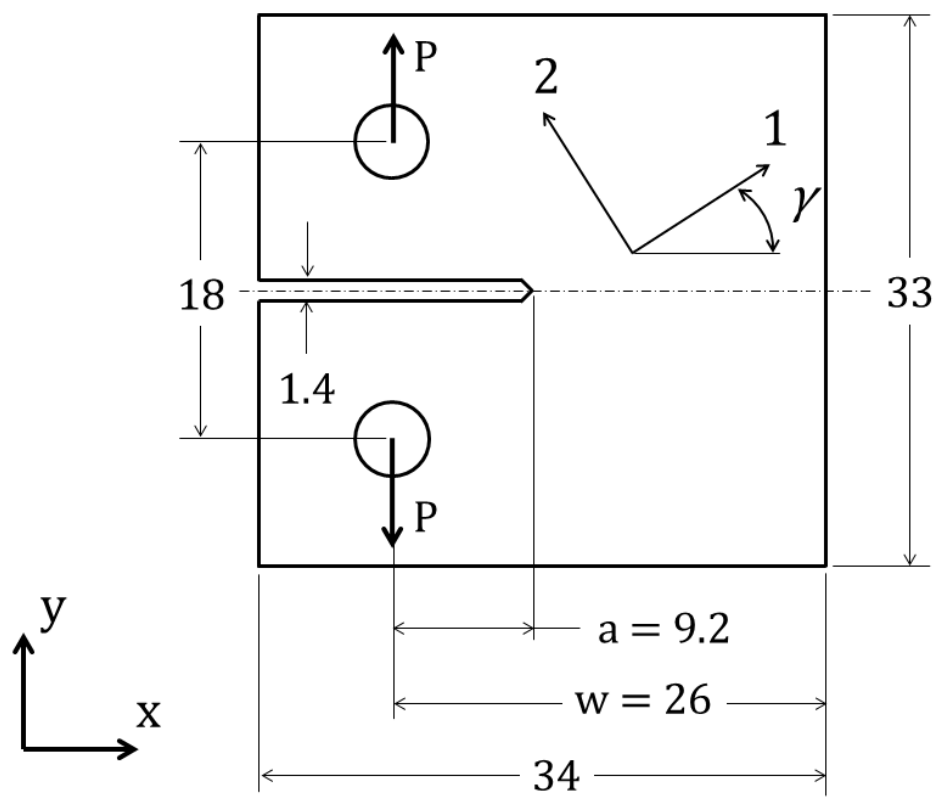

Figure 7 The compact tension specimen - dimensions are in $\mathbf{m m}$.

The domain was discretised with uniform grids, $\delta=1 \mathrm{~mm}$ and $2 \mathrm{~mm}$, and $m=3,4,5$ and 6. It was also discretised with a non-uniform grid, $\delta=0.96 \mathrm{~mm}$. For this grid, $\Delta x$ was linearly decreased, from $0.32 \mathrm{~mm}$ (equivalent to $\mathrm{m}=3$ ) to $0.16 \mathrm{~mm}$ (equivalent to $\mathrm{m}=6$ ), from the left edge towards the right edge (Figure 7) and from the upper and lower edges towards the centre line (at where the notch is located). Hence, the grid is symmetrical about the notch. The specimen was loaded by applying $0.17 \mathrm{~mm}$ displacements, ramped up over $7 \mathrm{~ms}$, along the load lines shown in Figure 7. When each displacement was applied on one node 
approximately located at the centre of the loading pin, it was observed that the slope of the force-displacement curve was dependent on the grid spacing, $\Delta x$. To avoid this behaviour, the displacement was prescribed on a set of nodes located within a $1 \mathrm{~mm}$ diameter circle centred at the position of the loading pin. No-fail circular zones (Ha and Bobaru 2010), where bonds cannot break, with a $4 \mathrm{~mm}$ diameter (diameter of the loading pins) centred at the loading pins were defined to avoid crack nucleation in the vicinity of these nodes. The ramp duration was long enough to ensure that dynamic effects were negligible before fracture and that these effects did not influence the initiation of crack propagation. Figure 8 indicates that the kinetic energy of the specimen was negligible as compared with the elastic energy. This figure also shows that the total energy of the system, which is the sum of the elastic energy, kinetic energy and the energy dissipated due to bond rupture (damage energy), perfectly matches the external work. This confirms the numerical implementation of the theory since different types of energies were evaluated independently.

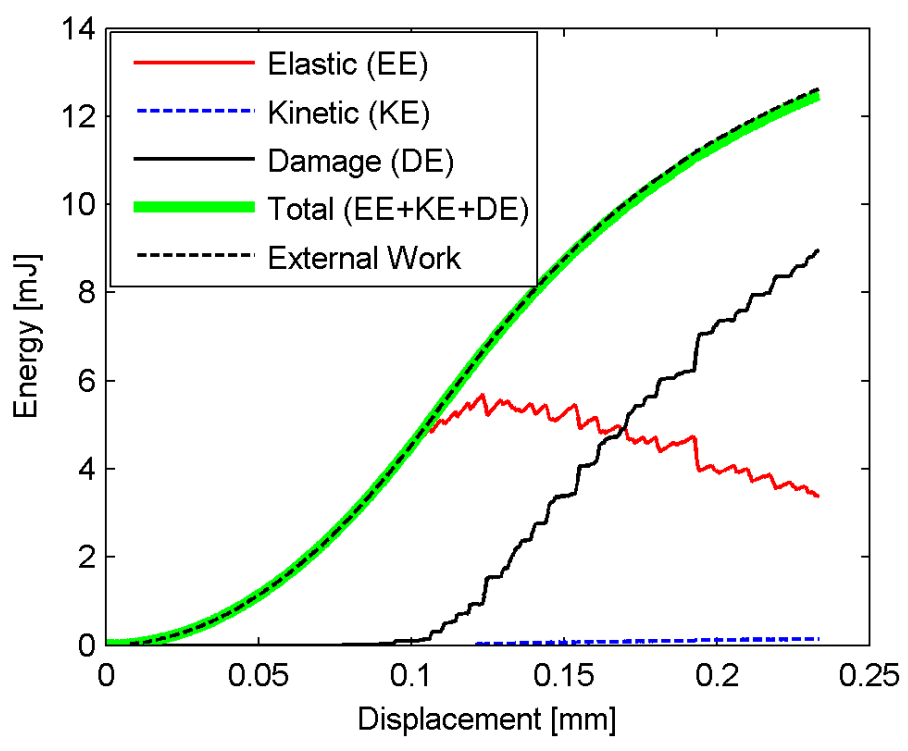

Figure 8 Different energies during the compact tension simulation $-\delta=1 \mathrm{~mm}$ and $\mathbf{m}=5$.

Typical load-displacement plots are shown in Figure 9. As can be seen, before damage initiates, the load increases linearly with displacement. The slope of this region of the loaddisplacement plot has been reported in Table 3 for all models. The difference between the reported values is marginal (less than 6\%), which confirms that the models have converged in the elastic region. The maximum damage propagation load, $P_{c}$, has also been reported in Table 3. As can be seen, when $m$ increases to $4, P_{c}$ decreases by up to $14 \%$. Using larger 
values of $\mathrm{m}$ has a negligible effect on $P_{c}$, particularly when $\delta=1 \mathrm{~mm}$, but it significantly increases the solution time (Table 2). For all models, except the model with a uniform grid $\delta$ $=2 \mathrm{~mm}$ and $m=3$, the difference between the predicted $P_{c}$ and that determined from the test (Behiri and Bonfield 1984), i.e. $98 \mathrm{~N}$, was less than 8\%. It should be mentioned that in (Behiri and Bonfield 1984) the following equation was used to determine the critical stress intensity factor:

$$
K_{I C 2}=\frac{P_{c}}{B w^{1 / 2}} Y
$$

where

$$
Y=29.6\left(\frac{a}{W}\right)^{1 / 2}-185.5\left(\frac{a}{w}\right)^{\frac{3}{2}}+655.7\left(\frac{a}{w}\right)^{\frac{5}{2}}-1017\left(\frac{a}{w}\right)^{\frac{7}{2}}+638.9\left(\frac{a}{w}\right)^{\frac{9}{2}}
$$

$a$ and $w$ are defined in Figure 7. The experimental value of $P_{c}=98 \mathrm{~N}$ was determined from eq. (60) for $K_{I c 2}=4.0 \mathrm{MPa} \cdot \mathrm{m}^{1 / 2}$ and $B=1 \mathrm{~mm}$.

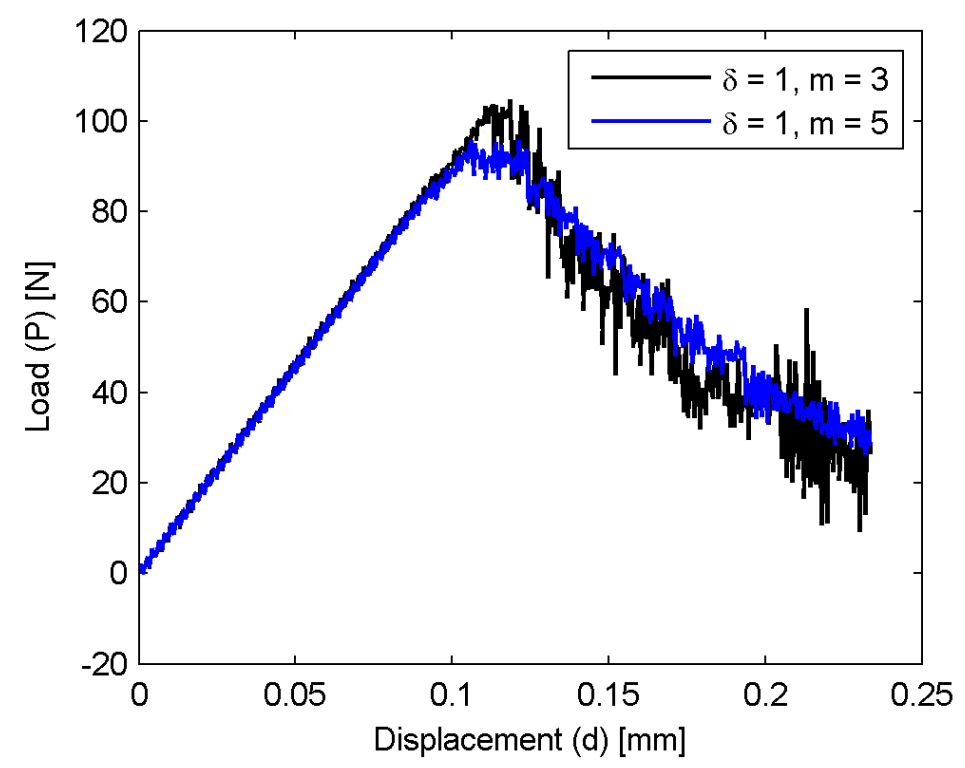

Figure 9 Typical load-displacement graphs for the cortical bone compact tension specimen. 
Table 3 Simulation results for the cortical bone compact tension specimen.

\begin{tabular}{|l|c|c|c|c|c|c|c|c|c|}
\hline$\delta[\mathrm{mm}]$ & \multicolumn{4}{|c|}{1} & \multicolumn{4}{c|}{2} & 1 \\
\hline $\mathrm{m}$ & 3 & 4 & 5 & 6 & 3 & 4 & 5 & 6 & Non-uniform grid \\
\hline Slope $^{\mathrm{a}}[\mathrm{N} / \mathrm{mm}]$ & 921 & 894 & 914 & 900 & 935 & 896 & 881 & 894 & 906 \\
\hline $\mathrm{P}_{\mathrm{c}}[\mathrm{N}]$ & 105 & 97 & 96 & 94 & 109 & 94 & 96 & 91 & 98 \\
\hline
\end{tabular}

a: the slope of the load-displacement curve in the elastic region.

Experimental observations (Behiri and Bonfield 1989) have shown that in cortical bone compact tension specimens with $\gamma>0^{\circ}$, the crack does not grow along the introduced notch. Instead, the fracture path is approximately parallel to the anatomical axis of the bone. In fact, the crack chooses a path so that it requires minimum energy to propagate. As can be seen in Figure 10, the peridynamic material model accurately predicts the failure paths when $\gamma=60^{\circ}$ and $90^{\circ}$. As can be seen, when $\gamma=90^{\circ}$, the crack propagates towards one side of the initial crack surface. For models with uniform grids, this occurred without injecting any imperfections into the model. In (Ha and Bobaru 2011), the asymmetry in the crack propagation path for a perfectly symmetrical model was attributed to the order of summation in eq. (51). For any two nodes symmetrically located on either side of the crack, the neighbours are not listed in the same order in the computational program. This would cause different round-off errors when evaluating the sum in eq. (51), which breaks the symmetry of the solution. Obviously, the asymmetric crack propagation path in the experiments was a result of imperfections in the geometry, material and boundary conditions. For the model with a non-uniform grid, the differences in round-off errors were not enough to break the symmetry of the model about the notch. Hence, the notch was slightly $(0.02 \mathrm{~mm})$ moved towards the upper edge of the specimen to break the symmetry. 

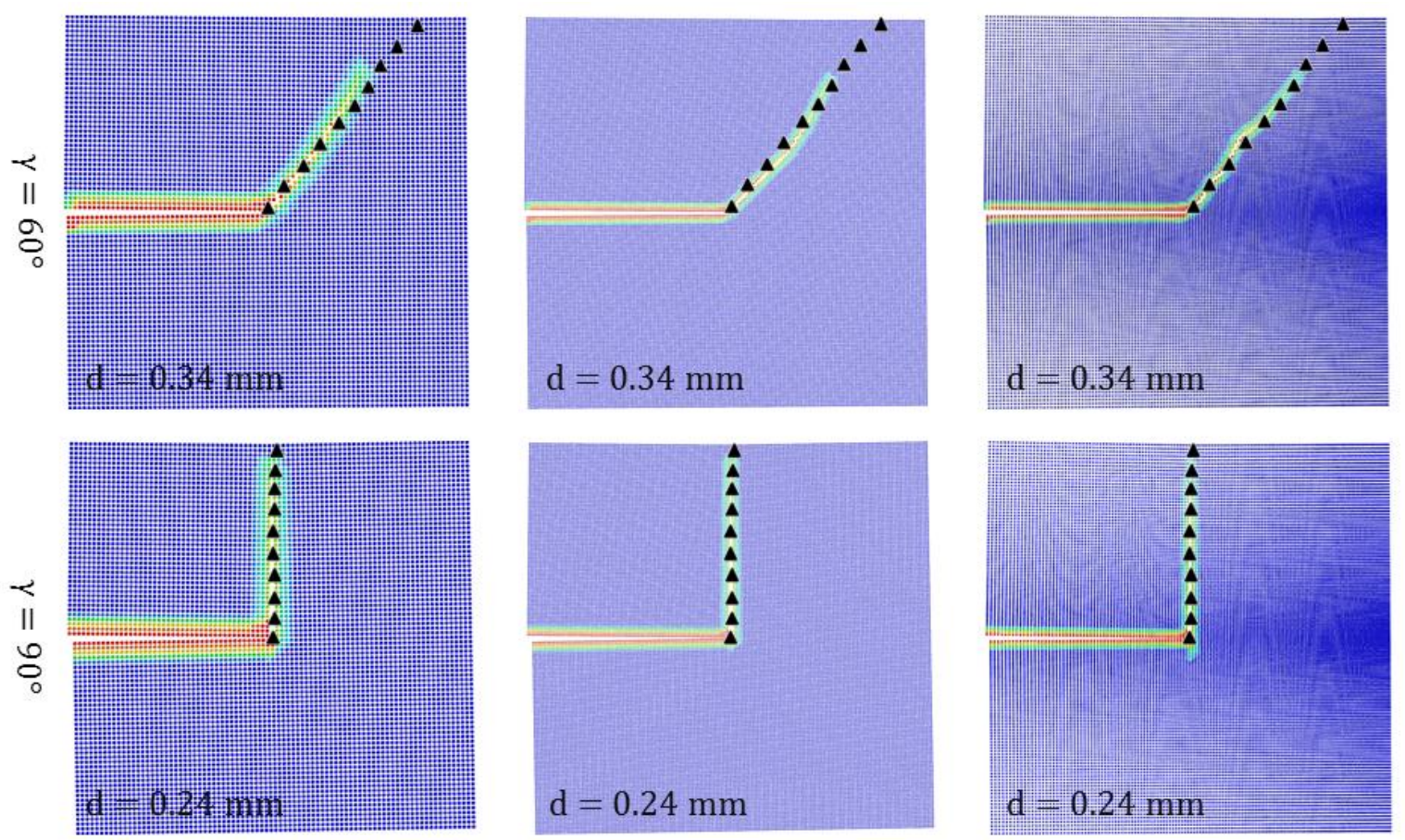

$$
\delta=2 \mathrm{~mm}, \mathrm{~m}=5
$$

$$
\delta=1 \mathrm{~mm}, \mathrm{~m}=5
$$

$\delta=1 \mathrm{~mm}$, non-uniform grid

\section{Figure 10 Experimental $(\Delta)$ and predicted crack propagation paths for the cortical bone compact tension specimen.}

\subsection{Dynamic fracture of polycrystalline microstructures}

In this section, the applicability of the peridynamic model to prediction of microfracture in polycrystalline materials has been demonstrated with an example. A polycrystalline material is composed of randomly oriented grains connected to each other at their interfaces. Microfracture of polycrystalline aggregates involves crack initiation, propagation of cracks between grains (intergranular fracture) and through grains (transgranular fracture), and crack branching and arrest. These complex phenomena are affected by random location, morphology and orientation of grains, and the ratio of the fracture toughness of the grain boundary $\left(G^{g b}\right)$ to that of the grain interior $\left(G^{\text {grain }}\right)$. In previous studies, the Boundary Element method and the Finite Element method were used to predict intergranular fracture (Espinosa and Zavattieri 2003a; Yousef et al. 2005; Sfantos and Aliabadi 2007; Benedetti and Aliabadi 2013a), and the FEM and particularly the X-FEM were used to predict inter/transgranular fracture and their interaction within a polycrystalline microstructure (Sukumar et al. 2003; Zhai et al. 2004). The peridynamic theory is also a suitable method for simulating dynamic brittle fracture in polycrystalline microstructures because it allows for crack nucleation and growth within and between grains and can predict crack branching without 
requiring any external criteria. Peridynamic predictions of inter/trans-granular fracture within a polycrystal with cubic symmetry (Benedetti and Aliabadi 2013b) have been presented in (Askari et al. 2008). The material model proposed in the current paper extends the capabilities of the bond-based peridynamic theory so that it can be used to model polycrystalline microstructures whose randomly oriented grains are transversely isotropic (hexagonal systems), such as alumina $\left(\mathrm{Al}_{2} \mathrm{O}_{3}\right)$.

The example presented in this section is an aggregate of 100 grains of alumina, within a square, subjected to dynamic tension. The average grain size was ASTM G $=5$ (E112-10 2010), which corresponds to an average grain area of $4032 \mu^{2}$. To represent random morphology and location of grains, the microstructure was generated by employing the Voronoi tessellation method, available within the Multi-Parametric Toolbox for MATLAB (Herceg et al. 2013). The generator points were obtained by using a two dimensional quasirandom generator (Sfantos and Aliabadi 2007). Since the model was two dimensional (plane strain with the 1 axis parallel to the axis of isotropy), one axis of the principal material frame (the 123 frame) of each grain was randomly selected and made coincident with the $\mathrm{z}$ axis of the global frame (the xyz frame). The other axes were rotated about the $\mathrm{z}$ axis by a randomly selected angle, $0^{\circ} \leq \gamma<360^{\circ}$, as shown in Figure 11. It is clear that this angle does not affect the mechanical response of the grain when $1 \equiv \mathrm{z}$.

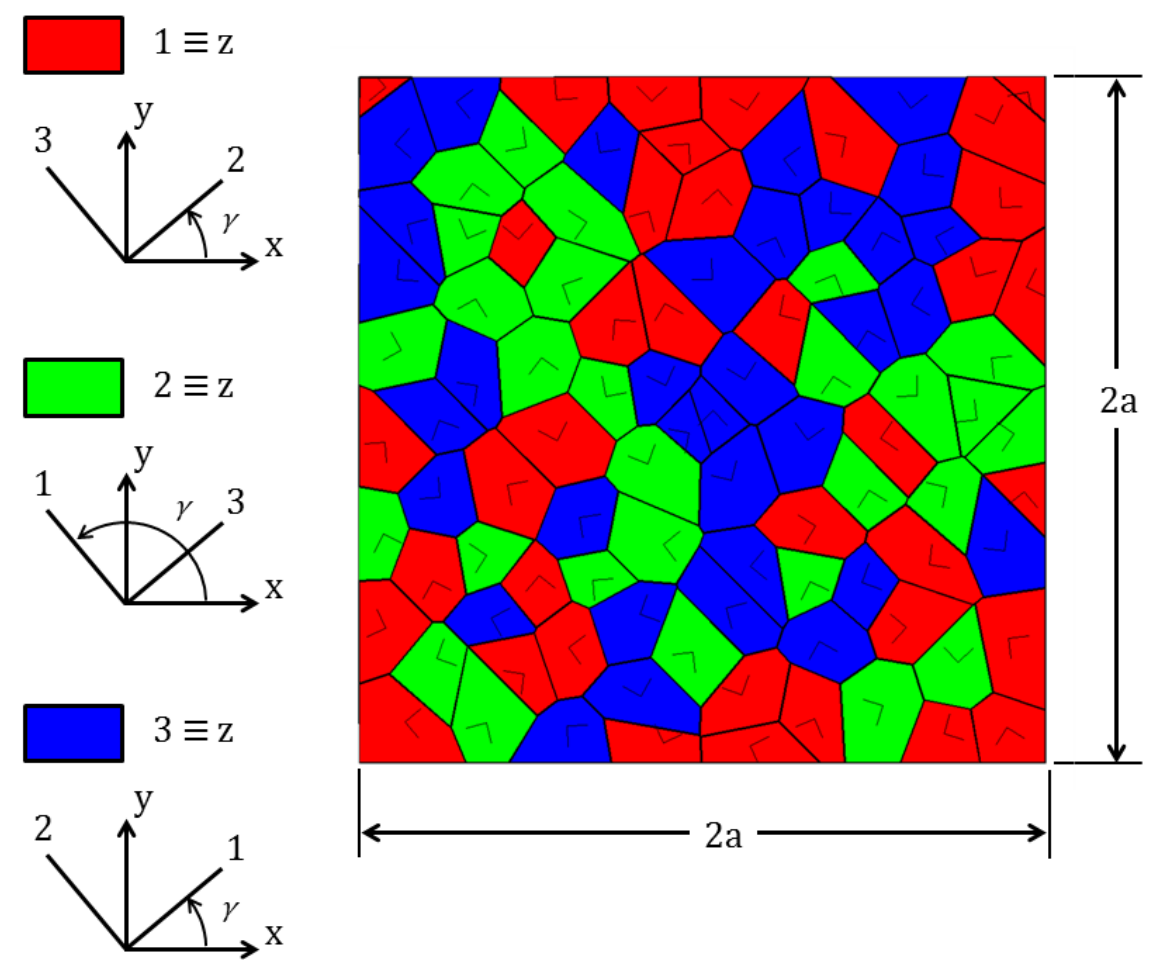

Figure 11 Random orientation of the principal material axes of the grains. 
For the alumina grains, the stiffness properties $C_{11}=563 \mathrm{GPa}$ and $C_{22}=465 \mathrm{GPa}$ were used (Espinosa and Zavattieri 2003b). The grain boundary fracture energy was assumed to be equal to the lattice fracture energy $G^{g b}=10 \mathrm{~J} / \mathrm{m}^{2}$ (Yousef et al. 2005). $G^{g b} / G^{\text {grain }}$ was varied $(0.1,0.5$ and 1.0$)$ in order to investigate its influence on the microfracture pattern and the failure load. The bonds that connect particles in two different grains, called the interface bonds, control the grain boundary behaviour. It was assumed that the properties of these bonds do not change with their orientation (isotropic). The stiffness constant of the interface bonds was determined using eq. (30), in which the average of $C_{11}$ and $C_{22}$ was inserted. To ensure that the correct amount of energy is dissipated when cracks propagate along the grain boundary, the critical stretch of the interface bonds was determined using eq. (40), in which $G^{g b}$ was inserted.

The specimen was discretised with a uniform grid, $\delta=10 \mu \mathrm{m}$ and $\mathrm{m}=5$. If the origin of the global coordinate system is at the centre of the specimen, the boundary conditions were: $u_{x}(-a, 0, t)=u_{x}(a, 0, t)=0, \quad u_{y}(0,-a, t)=-V \cdot t \quad$ and $\quad u_{y}(0, a, t)=V \cdot t, \quad$ where $\mathbf{u}(x, y, t)$ is the displacement vector and $V$ is a constant velocity. The displacements were applied within a layer of $\delta$ thickness under the edge. Since one aim was to predict the macroscopic elastic moduli of the ceramic, $V$ and the loading duration, $0.1 \mathrm{~m} / \mathrm{s}$ and $6 \mu \mathrm{s}$ respectively, were chosen so that there were several reflections of the stress waves within the specimen prior to failure. Figure 12 shows that in the top and bottom regions, the relative displacement between the last layer of nodes with prescribed displacements (i.e. the layer of nodes immediately above the line $\mathrm{AA}^{\prime}$ and that immediately below the line $\mathrm{BB}^{\prime}$ ) and the adjacent layer of nodes without a prescribed displacement is significantly larger than the relative displacement between the nodes within the bulk of the material. As a result, the bonds that connect nodes on opposite sides of the $\mathrm{AA}^{\prime}$ and $\mathrm{BB}^{\prime}$ lines break early during the simulation, leading to crack initiation at these zones. To avoid this, these bonds were not allowed to break during the simulation. A significant reduction in $V$ may also avoid premature fracture at the boundaries but the loading duration has to be increased drastically, which renders the simulation time unreasonable. 


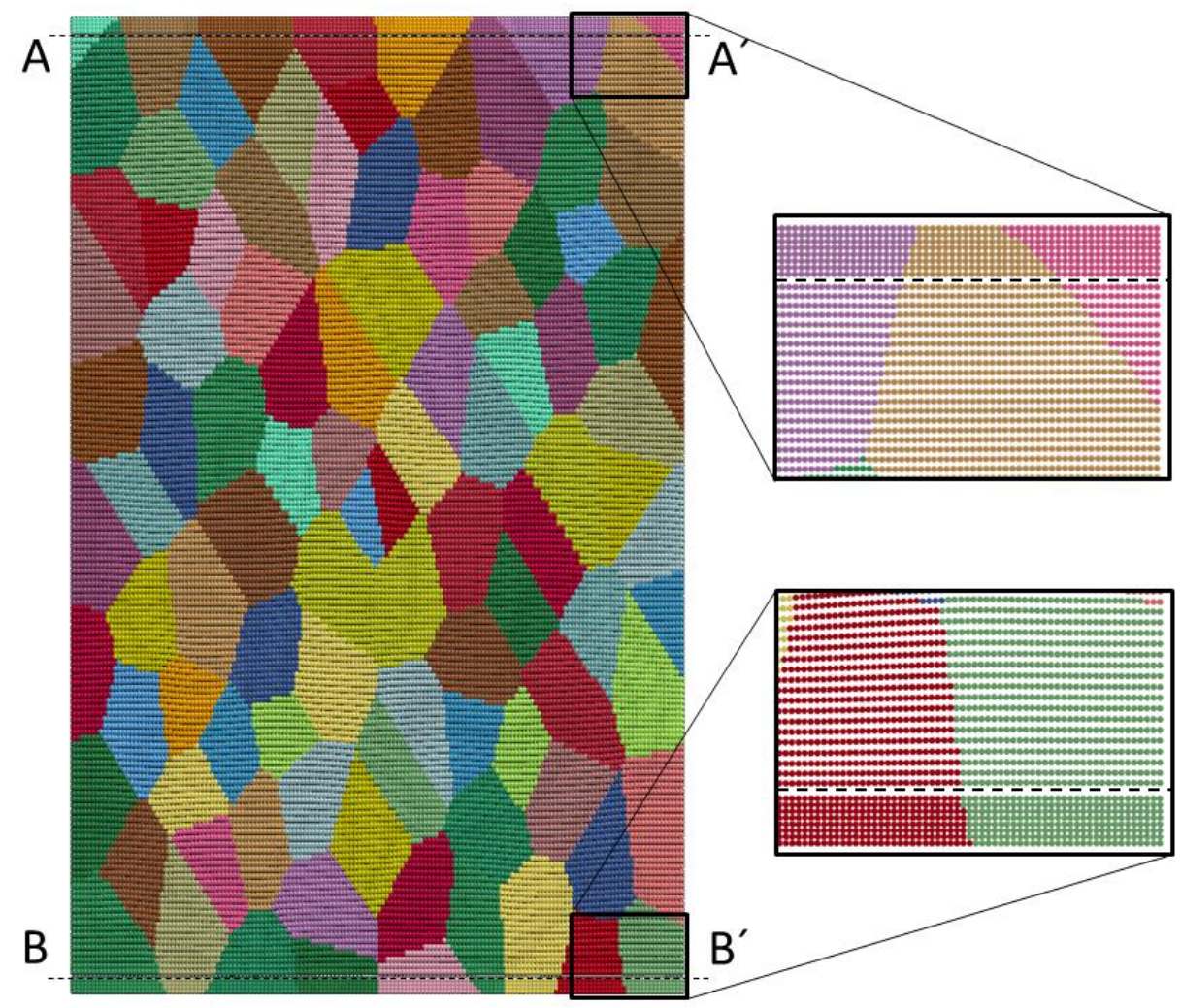

Figure 12 Displacement of the specimen exaggerated by a factor of 1000. Ramp displacements are applied on the nodes above the line $\mathrm{AA}^{\prime}$ and those below the line $\mathrm{BB}^{\prime}$.

The average stresses on the specimen were determined by averaging the tractions:

$$
\begin{aligned}
& \bar{\sigma}_{x}(t)=\frac{t_{x}(-a, 0, t)+t_{x}(a, 0, t)}{2}, \\
& \bar{\sigma}_{y}(t)=\frac{t_{y}(0,-a, t)+t_{y}(0, a, t)}{2} .
\end{aligned}
$$

Each traction was obtained by summing the reaction forces measured at the nodes on which the displacement was prescribed and dividing the resulting value by $2 a$. First degree polynomials were fitted to $\bar{\sigma}_{x}\left(t<t_{0}\right) v s . \bar{\varepsilon}_{y}\left(t<t_{0}\right)$ and $\bar{\sigma}_{y}\left(t<t_{0}\right) v s . \bar{\varepsilon}_{y}\left(t<t_{0}\right)$ plots, where $t_{0}$ is the time corresponding to damage initiation and $\bar{\varepsilon}_{y}(t)=V \cdot t / 2 a$. The slopes of these polynomials are respectively $C_{12}$ and $C_{11}=C_{22}$. Effective moduli of the microstructure, namely Young's modulus and Poisson's ratio, were determined by solving the following equations (Jones 1999) simultaneously: 


$$
\begin{aligned}
C_{11} & =\frac{(1-v) E}{(1+v)(1-2 v)}, \\
C_{12} & =\frac{v E}{(1+v)(1-2 v)} .
\end{aligned}
$$

In order to consider the influence of random location, morphology and orientation of grains on the effective moduli, ten microstructures were randomly generated, as described above, and loaded up to failure. Young's modulus and Poisson's ratio obtained from the simulation results were $393 \pm 3 \mathrm{GPa}$ and $0.235 \pm 0.001$, respectively, which are in good agreement with the values reported for a very low porosity alumina (at least $99.6 \% \mathrm{Al}_{2} \mathrm{O}_{3}$ ), $380-410 \mathrm{GPa}$ and $0.24-0.27$ (Auerkari 1996).

The microfracture patterns predicted for three different $G^{g b} / G^{\text {grain }}$ ratios are shown in Figure 13. For these cases, damage initiates at the interior of the specimen, near its centre. Subsequently, the damage propagates towards the left and right edges in the form of a crack. Both tips of the crack branch into several cracks, which some of them join as they propagate towards the edges. As can be seen in Figure 13, when the grain boundary is significantly weaker than the grain interior, the fracture mode is transgranular. For $G^{g b} / G^{\text {grain }}=0.5$, the model predicts a combination of the inergranular and transgranular fracture modes, with the former being dominant. When $G^{g b}=G^{\text {grain }}$, cracks propagate through the grains. These results agree well with the predictions made by using a peridynamic model (Askari et al. 2008) and also the predictions made by using an X-FEM model of a polycrystalline microstructure (Sukumar et al. 2003). The X-FEM model, however, predicted a single crack path while the peridynamic models predicted crack branching. It should be noted that in contrast to the X-FEM method (Belytschko et al. 2003), the peridynamic theory does not require any special criterion for crack branching. 


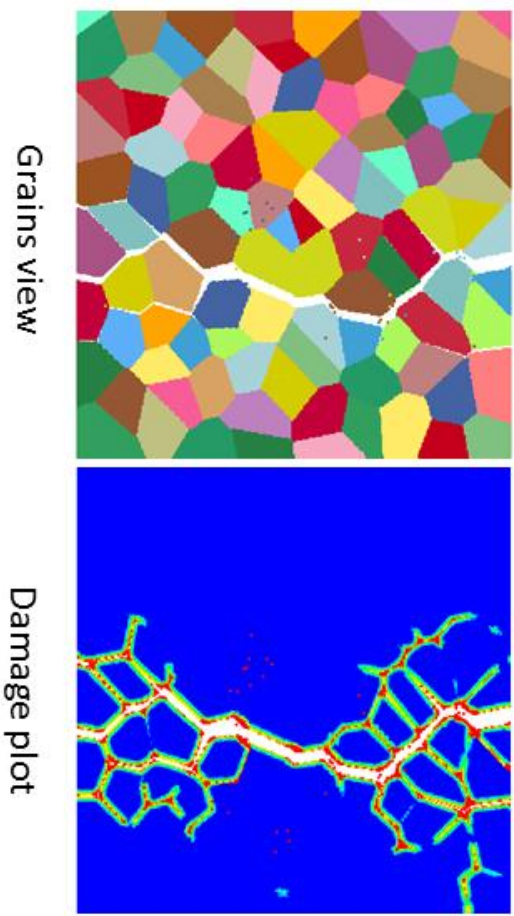

$\mathrm{G}^{\mathrm{gb}} / \mathrm{G}^{\text {grain }}=0.1$
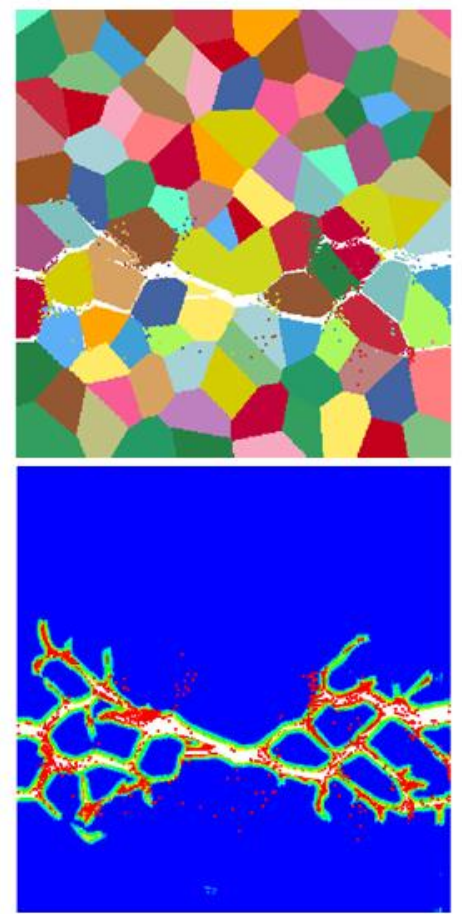

$\mathrm{G}^{\mathrm{gb}} / \mathrm{G}^{\text {grain }}=0.5$
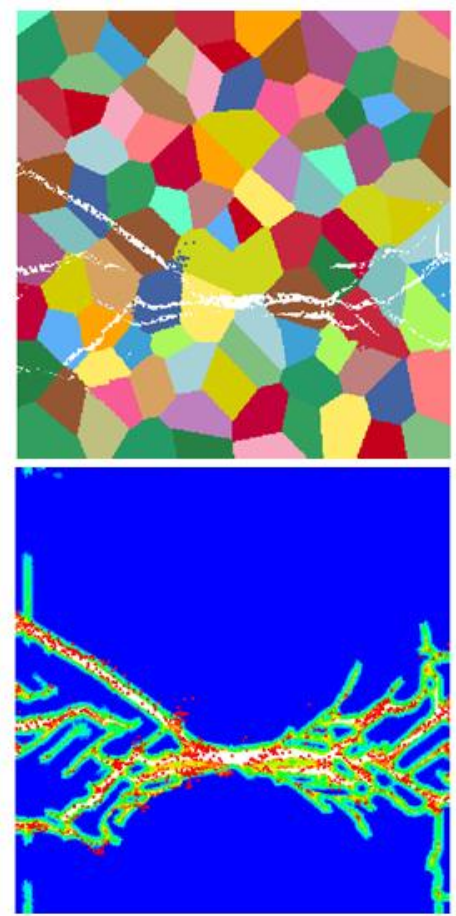

$\mathrm{G}^{\mathrm{gb}} / \mathrm{G}^{\text {grain }}=1$

Figure 13 Dependency of crack patterns in an alumina microstructure on the fracture toughness ratio. Displacements are exaggerated by a factor of 20.

The plot of the reaction force measured at $(x, y)=(0, a)$ vs. displacement is shown in Figure 14. The response of the microstructures is linear up to failure. For all $G^{g b} / G^{\text {grain }}$ ratios, the failure force and the corresponding displacement are almost equal. However, the bar plot of damage energies in Figure 14 indicates that when $G^{g b} / G^{\text {grain }}=0.1$, the fracture process dissipated less energy compared with the other two cases. These simulations, together with further studies on the influence of morphology, random location and size of grains on the fracture response of polycrystalline microstructures, can provide insight into microscale behaviour of polycrystalline materials and help optimise them for the failure load and energy absorption. 

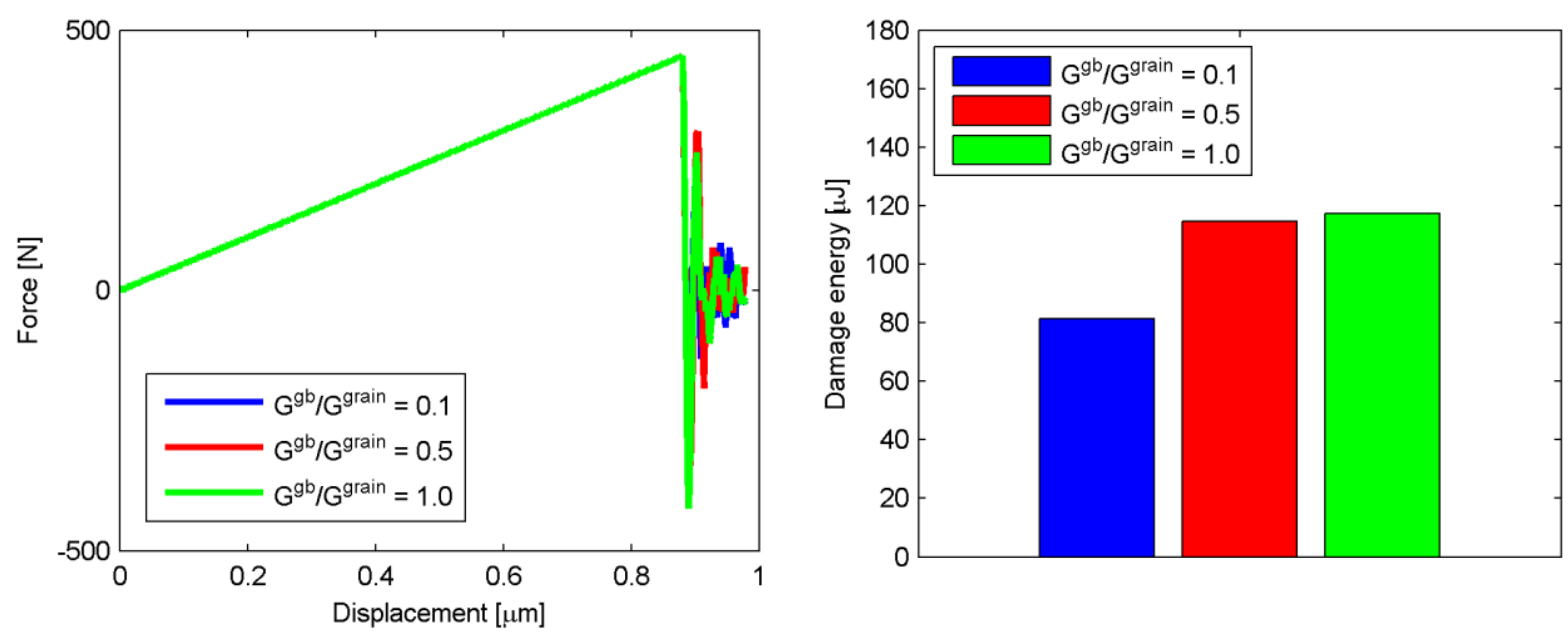

Figure 14 The force-displacement and damage energy plots of the alumina microstructure under tension.

\section{Conclusions}

A new peridynamic material model has been proposed for the analysis of fracture propagation in anisotropic materials under plane-stress and plane-strain conditions. The spherical harmonic expansion has been used to define the dependence of the bond stiffness constant and the bond critical stretch on bond orientation. This has simplified the evaluation of the integrals that appear in the equations relating engineering material constants and constants of the peridynamic model. Furthermore, since the bond stiffness constant and the critical stretch are continuous functions of bond orientation, the model can be used with any discretisation of the domain and any orientation of the principal material axes. Without compromising its advantages over previous models, this model can be easily extended to 3D.

Several fracture problems in different length scales have been analysed with the proposed approach. These problems are of technological or clinical importance. The results show very good quantitative agreement with analytical calculations and experimental results. Converged solutions have been obtained with reasonable mesh refinement and relatively small values of $m$ (the ratio between the horizon and the grid spacing, i.e. $\delta / \Delta x$ ). For a highly orthotropic composite lamina, $m=4$ has been sufficient to accurately predict the elastic response. For the compact bone with $G_{I c 1} / G_{I c 2}>2$, the fracture behaviour has been well predicted with a uniform grid with $m=4$ and also with a non-uniform grid. The fracture analysis of the alumina ceramic further illustrates the capabilities of the model in predicting complex fracture phenomena, such as intergranular and transgranular crack propagation in 
heterogeneous brittle microstructures. The proposed material model can be readily used to study the influence of pre-existing cracks, voids and inclusions on microfracture of ceramics.

\section{Acknowledgment}

This research was supported by a funding from the Engineering and Physical Sciences Research Council (EPSRC) and the Defence Science and Technology Laboratory (DSTL) under the project "Improving Survivability of Structures to Impact and Blast Loadings" EP/G042861/1.

\section{References}

Aliabadi, M. and P. Sollero (1998). "Crack growth analysis in homogeneous orthotropic laminates." Composites science and technology 58(10): 1697-1703.

Askari, E., F. Bobaru, R. Lehoucq, M. Parks, S. Silling and O. Weckner (2008). "Peridynamics for multiscale materials modeling." Journal of Physics: Conference Series 125: 012078.

Auerkari, P. (1996). Mechanical and physical properties of engineering alumina ceramics, VTT Technical Research Center of Finland.

Behiri, J. and W. Bonfield (1984). "Fracture mechanics of bone - the effects of density, specimen thickness and crack velocity on longitudinal fracture." Journal of biomechanics 17(1): 25-34.

Behiri, J. and W. Bonfield (1989). "Orientation dependence of the fracture mechanics of cortical bone." Journal of biomechanics 22(8): 863-872.

Belytschko, T., H. Chen, J. Xu and G. Zi (2003). "Dynamic crack propagation based on loss of hyperbolicity and a new discontinuous enrichment." International Journal for Numerical Methods in Engineering 58(12): 1873-1905.

Benedetti, I. and M. Aliabadi (2013a). "A three-dimensional cohesive-frictional grain-boundary micromechanical model for intergranular degradation and failure in polycrystalline materials." Computer Methods in Applied Mechanics and Engineering 265: 36-62.

Benedetti, I. and M. Aliabadi (2013b). "A three-dimensional grain boundary formulation for microstructural modeling of polycrystalline materials." Computational Materials Science 67: 249-260.

Bobaru, F. and W. Hu (2012). "The meaning, selection, and use of the peridynamic horizon and its relation to crack branching in brittle materials." International Journal of Fracture 176(2): 215222.

Bobaru, F., M. Yang, L. F. Alves, S. A. Silling, E. Askari and J. Xu (2009). "Convergence, adaptive refinement, and scaling in 1D peridynamics." International Journal for Numerical Methods in Engineering 77(6): 852-877.

Bonfield, W. (1987). "Advances in the fracture mechanics of cortical bone." Journal of biomechanics 20(11): 1071-1081.

Boone, T. J., P. A. Wawrzynek and A. R. Ingraffea (1987). "Finite element modelling of fracture propagation in orthotropic materials." Engineering Fracture Mechanics 26(2): 185-201.

Chen, C. S., E. Pan and B. Amadei (1998). "Fracture mechanics analysis of cracked discs of anisotropic rock using the boundary element method." International Journal of Rock Mechanics and Mining Sciences 35(2): 195-218.

Cook, R., D. Malkus, M. Plesha and W. RJ (2002). Concept and Application of Finite Element Analysis, John Wiley B Sons, INC, 719pp.

Donadon, M. V., B. G. Falzon, L. Iannucci and J. M. Hodgkinson (2007). "Intralaminar toughness characterisation of unbalanced hybrid plain weave laminates." Composites Part A: Applied Science and Manufacturing 38(6): 1597-1611.

E112-10, A. (2010). Standard Test Methods for Determining Average Grain Size, ASTM International. 
Espinosa, H. D. and P. D. Zavattieri (2003a). "A grain level model for the study of failure initiation and evolution in polycrystalline brittle materials. Part I: Theory and numerical implementation." Mechanics of Materials 35(3): 333-364.

Espinosa, H. D. and P. D. Zavattieri (2003b). "A grain level model for the study of failure initiation and evolution in polycrystalline brittle materials. Part II: numerical examples." Mechanics of Materials 35(3): 365-394.

Gerstle, W., N. Sau and S. Silling (2005). Peridynamic modeling of plain and reinforced concrete structures. 18th International Conference on Structural Mechanics in Reactor Technology, China.

Ha, Y. D. and F. Bobaru (2010). "Studies of dynamic crack propagation and crack branching with peridynamics." International Journal of Fracture 162(1-2): 229-244.

Ha, Y. D. and F. Bobaru (2011). "Characteristics of dynamic brittle fracture captured with peridynamics." Engineering Fracture Mechanics 78(6): 1156-1168.

Herceg, M., M. Kvasnica, C. N. Jones and M. Morari (2013). Multi-Parametric Toolbox 3.0. European Control Conference, Switzerland.

Hu, W., Y. D. Ha and F. Bobaru (2011). "Modeling dynamic fracture and damage in a fiberreinforced composite lamina with peridynamics." International Journal for Multiscale Computational Engineering 9(6).

Hu, W., Y. D. Ha and F. Bobaru (2012). "Peridynamic model for dynamic fracture in unidirectional fiber-reinforced composites." Computer Methods in Applied Mechanics and Engineering 217220: $247-261$.

Jones, R. M. (1999). Mechanics of composite materials, Taylor \& Francis, Inc.

Ke, C. C., C. S. Chen, C. Y. Ku and C. H. Chen (2009). "Modeling crack propagation path of anisotropic rocks using boundary element method." International Journal for Numerical and Analytical Methods in Geomechanics 33(9): 1227-1253.

Laffan, M., S. Pinho, P. Robinson and L. Iannucci (2010). "Measurement of the in situ ply fracture toughness associated with mode I fibre tensile failure in FRP. Part I: Data reduction." Composites science and technology 70(4): 606-613.

Livermore Software Technology Corporation (2012), LS-PrePost,

Macek, R. W. and S. A. Silling (2007). "Peridynamics via finite element analysis." Finite Elements in Analysis and Design 43(15): 1169-1178.

MacRobert, T. M. (1967). Spherical Harmonics. An elementary treatise on Harmonic Functions, Pergamon Press.

Motamedi, D. and S. Mohammadi (2010). "Dynamic crack propagation analysis of orthotropic media by the extended finite element method." International Journal of Fracture 161(1): 21-39.

Oterkus, E. and E. Madenci (2012). "Peridynamic analysis of fiber-reinforced composite materials." Journal of Mechanics of Materials and Structures 7(1): 45-84.

Parks, M. L., R. B. Lehoucq, S. J. Plimpton and S. A. Silling (2008). "Implementing peridynamics within a molecular dynamics code." Computer Physics Communications 179(11): 777-783.

Parks, M. L., P. Seleson, S. J. Plimpton, R. B. Lehoucq and S. A. Silling (2010), "Peridynamics with lammps: A user guide", Technical Report, SAND2010-5549, Sandia National Laboratories.

Seleson, P., M. Gunzburger and M. L. Parks (2013). "Interface problems in nonlocal diffusion and sharp transitions between local and nonlocal domains." Computer Methods in Applied Mechanics and Engineering 266: 185-204.

Sfantos, G. and M. Aliabadi (2007). "A boundary cohesive grain element formulation for modelling intergranular microfracture in polycrystalline brittle materials." International Journal for Numerical Methods in Engineering 69(8): 1590-1626.

Sih, G. C., P. Paris and G. Irwin (1965). "On cracks in rectilinearly anisotropic bodies." International Journal of Fracture Mechanics 1(3): 189-203.

Silling, S. A. (2000). "Reformulation of elasticity theory for discontinuities and long-range forces." Journal of the Mechanics and Physics of Solids 48(1): 175-209.

Silling, S. A. and E. Askari (2005). "A meshfree method based on the peridynamic model of solid mechanics." Computers \& Structures 83(17-18): 1526-1535.

Silling, S. A., M. Epton, O. Weckner, J. Xu and E. Askari (2007). "Peridynamic states and constitutive modeling." Journal of Elasticity 88(2): 151-184. 
Silling, S. A., M. Zimmermann and R. Abeyaratne (2003). "Deformation of a peridynamic bar." Journal of Elasticity 73(1-3): 173-190.

Sukumar, N., D. Srolovitz, T. Baker and J. H. Prévost (2003). "Brittle fracture in polycrystalline microstructures with the extended finite element method." International Journal for Numerical Methods in Engineering 56(14): 2015-2037.

Toribio, J. and F. Ayaso (2003). "Anisotropic fracture behaviour of cold drawn steel: a materials science approach." Materials Science and Engineering: A 343(1): 265-272.

Van-Buskirk, W. C., S. C. Cowin and R. N. Ward (1981). "Ultrasonic measurement of orthotropic elastic constants of bovine femoral bone." Journal of biomechanical engineering 103: 67-72.

Wolfram Research, Inc. (2010), Mathematica, Version 8.0, Champaign, Illinois.

Xu, J. F., A. Askari, O. Weckner and S. Silling (2008). "Peridynamic analysis of impact damage in composite laminates." Journal of Aerospace Engineering 21(3): 187-194.

Yousef, S. G., J. Rödel, E. Fuller, A. Zimmermann and B. El-Dasher (2005). "Microcrack evolution in alumina ceramics: experiment and simulation." Journal of the American Ceramic Society 88(10): 2809-2816.

Zhai, J., V. Tomar and M. Zhou (2004). "Micromechanical simulation of dynamic fracture using the cohesive finite element method." Transactions-American society of mechanical engineers Journal of Engineering Materials and Technology 126(2): 179-191. 